\title{
Estudio de los factores determinantes de la deserción en el sistema universitario chileno
}

\author{
Tomás Larroucau
}

Universidad de Chile

\section{Resumen}

Este estudio tiene como objetivo identificar y cuantificar los factores determinantes de la probabilidad de deserción del sistema universitario chileno. La metodología utilizada considera un modelo econométrico estático de tres ecuaciones que pretende corregir, simultáneamente, la posible endogeneidad presente en la selección de muestra y en la obtención de las ayudas estudiantiles, diferenciando la deserción temporal de la definitiva y analizando posibles efectos heterogéneos entre áreas de carrera y tipos de universidad. Los resultados muestran que la deserción universitaria es un fenómeno multicausal, influida por factores de habilidad individual del estudiante, socioeconómicos y demográficos, pero también factores relacionados con la calidad del establecimiento de enseñanza media, de la carrera y de la universidad a la que accede. Las características previas al ingreso del estudiante a la universidad son más relevantes que la habilidad medida por medio de la PSU. Además, se constata que la deserción universitaria es también un problema de financiamiento; estudiantes de mayores ingresos y aquellos que perciben una ayuda estudiantil presentan una probabilidad estadísticamente significativa menor de desertar del sistema. Por otro lado, se encuentra que la calidad de la universidad y el tipo de carrera a la cual se accede son factores también importantes ya que existen indicios de heterogeneidad al interior del sistema.

Palabras clave: Modelos econométricos, deserción universitaria, sistema educacional.

\section{Study of the determinants of desertion in the chilean university system}

\begin{abstract}
This study identify and quantify the determinants of dropping out probability of the chilean univertsity system. The methodology considers a static econometric model of three equations to correct the possible endogeneity present in the sample selection and in the obtaining of student aid, differentiating the temporary and final desertion, and analyzing possible heterogeneous effects among career areas and university types. The results show that the college desertion is a multi-causal phenomenon, influenced by factors of individual student's ability, socioeconomic and demographic, but also factors related to the quality of the establishment of secondary education, career and university. The student's characteristics previous the university admission to the university are more important than the ability measured by the PSU. The university desertion is a funding problem; higher-income students and those who receive a student aid presented a statistically significant lower probability of deserting of the system. On the other hand, the quality of the university and the type of career are also important factors, because there is evidence of heterogeneity within the system.
\end{abstract}

Keywords: Econometric models, university desertion, education system.

*Dirección de correspondencia [Correspondence

address]: Tomás Larroucau, Universidad de Chile

E-mail: tlarroucau@gmail.com 


\section{Introducción}

El sistema de educación superior chileno ha sufrido grandes cambios en los últimos 30 años. A principios de la década de los 80 existían solo 8 universidades, la matrícula total bordeaba los 100.000 estudiantes y su financiamiento era principalmente de origen estatal. Para el año 2011, en cambio, existían 178 instituciones de educación superior: 60 universidades (25 pertenecientes al CRUCH y 35 universidades privadas no tradicionales), 45 institutos profesionales (IP) y 73 centros de formación técnica (CFT). Por otro lado, el financiamiento estatal cambió de foco, de subsidiar la oferta a subsidiar la demanda, para lo cual se crearon numerosas becas y créditos enfocados en los sectores más vulnerables del país. Finalmente, la matrícula del sistema completo superó el millón de estudiantes, significando un aumento sustancial en la cobertura neta de educación superior en la población.

Los aumentos en cobertura del sistema vinieron acompañados con bajas tasas de permanencia y retención. Por otro lado, se aprecia una gran heterogeneidad al interior del sistema universitario chileno, encontrándose diferencias muy elevadas en las tasas de deserción entre áreas de carrera y tipos de universidad (Centro de microdatos, 2008). Esto puede dar luces que existen problemas de deserción importantes en el sistema, los cuales no han sido abordados apropiadamente.

Comprender las causas de la deserción universitaria es de suma relevancia. En primer lugar, la deserción tiene un costo personal para el estudiante y su familia, ya que además de elevar los costos del proceso educativo del estudiante (llegando incluso a ser un costo hundido más que una inversión), incide fuertemente en la motivación del individuo, generando frustración y problemas psicosociales (González, 2005). En segundo lugar, porque se invierten numerosos recursos en becas y créditos universitarios que, finalmente, no terminan con la graduación del estudiante. En este contexto, es de vital importancia conocer los factores determinantes de la deserción universitaria, para de esta forma poder diseñar e implementar políticas que aumenten las tasas de retención y graduación de los estudiantes.

Dado lo anterior, el presente estudio se propone identificar y cuantificar los factores determinantes de la probabilidad de deserción del sistema universitario chileno. Para ello, se utiliza un modelo econométrico de tres ecuaciones, que pretende corregir la posible endogeneidad presente en la selección de muestra y en la obtención de las ayudas estudiantiles, simultáneamente. Además, se diferencia el fenómeno temporal del definitivo y se entregan indicios de los posibles efectos heterogéneos en las distintas áreas de carrera y tipos de universidad. La estimación de este modelo econométrico, se hace a partir de datos correspondientes a la cohorte de estudiantes que rindió la prueba SIMCE en el año 2003 y la Prueba de Selección Universitaria (PSU) en los años 2005 o 2006, siguiendo su permanencia en el sistema universitario hasta el año 2011.

\section{Deserción educacional}

El fenómeno de la deserción educacional ha sido ampliamente estudiado en la literatura internacional. Algunos estudios se centran en modelar el problema de la deserción de manera teórica, mientras que otros utilizan como base estos modelos, para encontrar las causas de la deserción en una población determinada utilizando distintas metodologías, ya sea cualitativas o cuantitativas.

Uno de los modelos teóricos es el de Tinto (1975), quien propone un modelo a nivel conceptual (Student Integration Model) que explica el proceso de deserción, por la interacción de elementos individuales, sociales e institucionales. Destacan como los factores más relevantes: las características familiares de los estudiantes, las expectativas educativas y el nivel de compromiso con metas y objetivos futuros. Estos factores también son destacados por otros autores, explicando que las actitudes positivas de los pares y padres disminuye la probabilidad de deserción del estudiante (Bank Slaving, R. \& Biddke, B., 1990). Pese a ser un modelo bastante exhaustivo, Tinto no considera factores externos distintos de los institucionales, como las tasas de desempleo por carrera o el nivel de ingreso esperado.

Otros modelos teóricos, como el Student Attrition Model de Bean (1980), ponen especial énfasis en factores externos del mercado laboral. Pese a ello, se sigue manteniendo la tesis de multicausalidad en el fenómeno, ya que también se le otorga importancia a factores explicativos como las expectativas del estudiante, el apoyo de los padres y el financiamiento de los estudios.

Finalmente, uno de los modelos teóricos más recientes es el College Choice Nexus Model, propuesto por (St.John et al., 2000). En este caso, el fenómeno de la deserción es modelado como un proceso que ocurre en tres etapas. Inicialmente el estudiante decide si accede o no a la universidad, donde los factores más relevantes son los socioeconómicos y los relacionados con la habilidad del estudiante. Luego, realizando un análisis de costo beneficio, el estu- 
diante elige la institución a la cual accederá y, finalmente, dependiendo de su rendimiento académico y de la nueva información recibida del mercado laboral, decide si desertar o no. Este modelo plantea que el proceso de deserción no puede ser separado del de acceso o elección. Sin embargo, el análisis de la decisión de desertar como una respuesta racional del individuo en relación a nueva información (similar al enfoque de capital humano propuesto por Becker (1964)), tanto económica como no económica, puede relegar a segundo plano las características intrínsecas del estudiante (como el background familiar), las cuales empíricamente se han posicionado como factores determinantes de la deserción educacional.

En relación con los tipos de deserción educacional, en la literatura se establecen ciertas distinciones. Tinto (1975) diferencia el proceso de carácter temporal o parcial (stopout), del permanente o definitivo (dropout) y lo divide también, si este es de carácter voluntario o forzoso. El autor conceptualiza la deserción universitaria como el proceso de abandono voluntario o forzoso de la carrera en que se matricula el estudiante y esta puede ser influenciada de manera positiva o negativa por factores internos o externos a él.

Dentro de los estudios empíricos a nivel internacional destacan los modelos econométricos (estáti$\cos$ ), los cuales miden el efecto en la variable dependiente de una serie de factores en un tiempo específico. Dentro de los modelos econométricos, los más utilizados son los modelos probabilísticos (Barrios F., 2011), donde se mide la probabilidad de desertar, controlando por una serie de variables explicativas, obteniendo así su contribución marginal en la ecuación de interés (Stratton et al., 2008; Cameron, 2009; Melguizo et al., 2011). Siguiendo esta línea, algunos estudios empíricos como el de Stratton, O‘Toole y Wetzel (2008) muestran cómo el no diferenciar la deserción temporal de la permanente puede llevar a la obtención de coeficientes sesgados, debido a que existen diferencias significativas entre sus factores determinantes. Cabe notar que el foco predominante en estos estudios es analizar el efecto de las ayudas estudiantiles sobre la deserción.

Últimamente se han utilizado también los modelos dinámicos, como modelos de duración (Lassibille y Navarro Gómez, 2008; DesJardins et al., 2006). En ellos, se testea la hipótesis de la dependencia del fenómeno en el tiempo, siendo un proceso que se ve afectado por la irrupción de determinados factores a lo largo del ciclo de evaluación. En el estudio de Lassibille y Navarro Gómez (2008) se analiza una universidad en España, siguiendo una cohorte por 8 años. En él, se demuestra que estudiantes con menos habilidades, menos motivación, que entran más tarde a la educación superior, poseen una mayor probabilidad de desertar. Por otro lado, se realizan modelos para los distintos tipos de facultades o institutos (University faculties, Higher technical schools y University schools), identificando los tipos de programa de estudios. Finalmente encuentra que existe heterogeneidad entre programas de estudios y tipos de universidad en relación a la deserción universitaria.

Pese a que algunos autores critican la utilización de los modelos estáticos (Lassibille y Navarro Gómez, 2008), el fenómeno de la deserción resulta predominante en los dos primeros años de la educación universitaria, por lo que las condiciones de acceso parecieran ser las más relevantes.

La investigación de la deserción universitaria a nivel nacional es bastante nutrida, encontrándose estudios cualitativos, estudios de análisis estadísticos y otros que utilizan modelos econométricos para estimar los factores determinantes del fenómeno.

Los estudios cualitativos son más bien de carácter conceptual, como los de Himmel (2002), Díaz (2008) y Donoso y Schiefelbein (2007). En ellos se entrega una buena categorización de los enfoques que explican la deserción. Uno de los aportes significativos en el caso de Himmel, es definir el concepto de deserción de forma unívoca. Argumenta que se debe adoptar un concepto único para poder comparar las distintas investigaciones y define en este caso la deserción como: "abandono prematuro de un programa de estudios antes de alcanzar el título o grado y considera un tiempo suficientemente largo como para descartar la posibilidad de que el estudiante se reincorpore" (Himmel, 2002). En esta línea, a la deserción del sistema educativo (y no solo del plan de estudio) Himmel la llama "abandono definitivo".

A nivel cuantitativo se encuentran estudios de análisis estadístico, pero muy pocos utilizan modelos econométricos. En relación a los de estadística descriptiva, destacan los realizados por González y Uribe (2002), González (2005) por el Centro de microdatos (2008). En el estudio de González (2005), se afirma que los factores institucionales y aspectos sociales son causantes de la deserción a nivel universitario, coincidiendo con la evidencia internacional respecto a la multicausalidad de los factores. Por otro lado, en el estudio realizado por Centro de microdatos (2008), se encuentran los problemas vocacionales, la situación económica de sus familias y el rendimiento académico de los estudiantes, como los principales factores determinantes de la deserción universitaria. En este estudio, se entrega además, estadística de las tasas de deserción por tipo de institución y carrera, evidenciándose dife- 
rencias sustanciales en ellas.

En relación con los estudios nacionales que utilizan modelos econométricos para medir la deserción universitaria a nivel general, hasta la fecha de revisión de literatura, se encontraron 5: Los estudios de Acuña et al. (2010), Barrios F. (2011),MINEDUC (2012), los cuales utilizan modelos probabilísticos del tipo logit o probit (con corrección de muestra en el caso de Acuña, Macovek y Mizala), los estudios de Solis (2011) e Intelis y Verde (2012) que utilizan metodología RD y el estudio de Rau et al. (2012) en el cual utilizan un modelo de elección condicionado más complejo. De estos estudios, se revisó en mayor detalle el de Barrios F. (2011) y el desarrollado por Acuña et al. (2010), debido a que son estudios probabilísticos similares a los que se encuentran en la literatura internacional. En el primero, se utiliza un modelo logit para estimar la probabilidad de deserción del sistema universitario, siguiendo un enfoque similar al de Stratton et al. (2008). Se analiza con gran detalle el efecto de las ayudas estudiantiles sobre la deserción universitaria, encontrándose que las ayudas estudiantiles disminuyen fuertemente la deserción universitaria, sin embargo, existe heterogeneidad en la respuesta de estas, en función del nivel de ingreso del estudiante. En el segundo estudio, se utiliza un probit con selección de Heckman, para medir la probabilidad de deserción del sistema, corregida por una ecuación de acceso. En este estudio se encuentra que: la habilidad, medida en base a la prueba PSU y el ranking de enseñanza media; el background familiar, medido por el nivel educacional de los padres y la cantidad de hermanos en la educación superior; y la calidad del establecimiento de origen, medida por el quintil del establecimiento de origen en la prueba SIMCE, son los factores más significativos en explicar la deserción de la educación superior. Ambos estudios son un avance significativo para comprender el fenómeno de la deserción universitaria, sin embargo, no diferencian entre la deserción temporal y la permanente o definitiva, pudiendo producirse sesgos en la estimación.

En relación con los estudios realizados por medio de modelos econométricos, tanto a nivel nacional como internacional, se han evidenciado problemas de endogeneidad en la estimación de los factores determinantes de la deserción. Por un lado, Acuña et al. (2010) plantean el posible sesgo al no corregir la estimación por medio de una ecuación de selección, problema conocido en la literatura como selección de muestra Heckman (1979). Este sesgo se produciría debido a que la población que accede al sistema no puede considerarse como una muestra aleatoria de la población potencial. Además del problema de selección de muestra, numerosos estudios evidencian el problema de endogeneidad presente en las ayudas estudiantiles, ya sea para estudiar el acceso o la deserción del sistema universitario (Cameron, 2009; Meneses y Blanco, 2010; Melguizo et al., 2011). Sesgo producido principalmente por la selección no aleatoria para obtener una ayuda estudiantil.

Cabe notar que existen dos estudios recientes: uno desarrollado por Verónica Santelices y el otro por Ricardo Paredes, en los cuales se estiman los factores determinantes de la deserción por medio de modelos econométricos y se intenta corregir la endogeneidad presente en las ayudas estudiantiles utilizando metodología de Matching y de RD. Estos estudios, aún no publicados a la fecha de revisión de literatura, ponen su foco principal en analizar las diferencias entre el tipo de ayuda que recibe el estudiante, encontrando que los créditos disminuyen en mayor proporción la probabilidad de desertar en comparación a las becas.

\section{Metodología}

\section{Modelo de categorías de deserción}

El presente estudio propone la utilización de modelos econométricos para cuantificar el efecto marginal de distintas variables sobre la probabilidad de deserción del sistema universitario. Para ello, se tuvo en consideración el modelo teórico propuesto por St.John et al. (2000), al modelar el proceso en etapas e incluir variables relacionadas con el mercado laboral; al de Tinto (1975), al considerar la interacción entre el estudiante y la institución a la cual accede, y a diversos estudios empíricos que develan la importancia de incluir ciertas variables en la especificación.

Se controlará la posible existencia de heterogeneidad en el fenómeno, distinguiendo entre tipos de universidad y áreas de carrera, evaluando el efecto sobre la deserción de carácter permanente, diferenciándola de la temporal. Por otro lado, para poder controlar el efecto sobre la población total (población potencial de estudiantes universitarios), se condicionará el fenómeno observado por el acceso a la educación superior, ya que la deserción es un fenómeno que se observa sólo en la población que accede al sistema. Además, se intentará corregir la endogeneidad presente en las ayudas estudiantiles, utilizando un enfoque de variable instrumental.

La decisión de desertar se puede modelar por medio de un ordered probit, donde la variable de interés puede ser categorizada de forma ordenada, en función de la utilidad que cada categoría le reporta al individuo. Si se define la variable de interés co- 
mo $y_{h}, h=1, \ldots, H$ la relación entre cada categoría sería: $y_{1}<y_{2}<$ ? $<y_{H}$

Si bien este modelamiento exige un orden de utilidad para cada categoría, no impone una diferencia cardinal específica entre ellas, por lo que la diferencia entre $y_{1}$ e $y_{2}$ no guarda relación alguna con la existente entre $y_{2}$ e $y_{3}$, y así sucesivamente. Sólo debe existir un orden entre las categorías.

Como la variable de interés es el tipo de deserción que realiza el estudiante, esta puede ser ordenada en forma creciente de utilidad, donde la opción que más utilidad le reporta al individuo es permanecer en el sistema universitario (Matrícula continua), luego el desertar temporalmente de él (Deserción temporal) y, finalmente, la opción que menos utilidad le reporta es el desertar definitivamente del sistema universitario (Deserción definitiva). Este ordenamiento resulta bastante lógico y se fundamenta en los altos costos que implica para el individuo la decisión de desertar, sumado a las elevadas tasas de retorno que presenta el permanecer en el sistema universitario en Chile (Meller y Lara, 2010).

Finalmente, el proceso puede ser modelado de la siguiente forma: $y_{i}^{*}=x_{i}^{\prime} \beta+\epsilon_{i}$

Donde $y_{i}^{*}$ representa la utilidad latente (o no directamente observable) de cada individuo $i, x_{i}^{\prime}$ es un vector de características individuales observables, $\beta$ es el vector de parámetros a estimar y $\epsilon_{i}$ es un término de error que sigue una distribución normal.

Luego la respuesta observada queda determinada por:

$y_{i h}=\left\{\begin{array}{l}y_{i 1}(\text { Deserción definitiva }) \text { si }-\infty<y_{i}^{*} \leq k_{1} \\ \left.y_{i 2} \text { (Deserción temporal }\right) \text { si } k_{1}<y_{i}^{*} \leq k_{2} \\ y_{i 3} \text { (Matrícula continua)si } k_{2}<y_{i}^{*} \leq \infty+\end{array}\right.$

Donde $y_{i h}$ es la respuesta observada del individuo $i$, eligiendo la categoría $h$ y $k_{1}, k_{2}$ representan parámetros umbrales para la utilidad latente.

Las variables observables $x_{i}^{*}$ indican las principales características del individuo $i$ que influyen sobre la probabilidad de realizar o escoger Deserción definitiva, Deserción temporal o Matrícula continua.

Claramente esta metodología restringe la posibilidad de estimar efectos que alteren el ordenamiento de las categorías. En este sentido, no se podrá estimar (por construcción), si para alguna variable determinada, la probabilidad de "escoger" Deserción temporal es mayor que la probabilidad de "escoger" las otras dos categorías (debido a que se construyó como una categoría intermedia en la función de utilidad), ni tampoco se podrá testear si los factores determinantes de la Deserción temporal son, a un nivel estadísticamente significativo, diferentes a los factores determinantes de la Deserción definitiva.

Por otro lado, el no considerar dentro del mismo proceso de decisión el realizar Deserción temporal, Deserción definitiva y Matrícula continua, llevaría a cometer el error de mezclar alguna de las tres categorías, o bien perder una gran cantidad de observaciones al excluir la Deserción temporal de la muestra (como en el caso de estimar un Probit simple para cada categoría). Por ello se prefirió el utilizar un Ordered Probit más que un Probit simple, pese a la restricción de ordenamiento, debido a que el foco de este estudio es estimar en mayor detalle la deserción definitiva del sistema universitario, considerando que el estudiante puede decidir también desertar temporalmente, pero no el analizar si existen diferencias estadísticamente significativas entre estos dos tipos de deserción.

Otro aspecto a destacar es que este modelo econométrico es de carácter estático, por lo que no es posible medir variaciones en ciertas variables de interés, lo que podría influir en la decisión de desertar de un estudiante. En este sentido, el modelo pretende medir cómo influyen ciertas variables de entrada (estáticas) en la probabilidad de deserción de un estudiante del sistema universitario.

\section{El problema de la selección de la muestra}

Para estimar los efectos de la deserción en la población total (población potencial de estudiantes universitarios), es necesario tener en cuenta que la muestra para la cual se observa la variable de interés, no necesariamente es representativa. Esto es conocido como el problema de la selección de muestra (Heckman, 1979).

En el problema de la selección de muestra, la variable de interés es observada solamente si una determinada condición de selección está presente $\left(S_{i}=1\right)$. Se observa si un individuo ha sido seleccionado $\left(S_{i}=1\right)$ o no $\left(S_{i}=0\right)$ en la muestra, pero sólo se observa la respuesta de la variable de interés si el individuo ha sido seleccionado. Utilizando un modelo de utilidad latente, como probit, esta situación se puede describir de la siguiente forma:

$$
S_{i}^{*}=z_{i}^{\prime} \gamma+\mu_{i}
$$

Donde $S_{i}^{*}$ es la utilidad latente del individuo $i$, 
$z_{i}^{\prime}$ es un vector de características individuales observables, $\gamma$ es el vector de parámetros a estimar y $\mu_{i}$ es un término de error que sigue una distribución normal. Luego la respuesta a la condición de selección queda determinada por:

$$
S_{i}=\left\{\begin{array}{l}
1 \text { si } S_{i}^{*}>0 \\
0 \text { si } S_{i}^{*}>0
\end{array} \quad \forall i=1 \ldots N\right.
$$

Para el caso de la deserción universitaria, la variable de interés se observa solamente en la población que accede a la universidad, por lo que el modelo completo sería representado por dos ecuaciones:

(1) $y_{i}^{*}=x_{i}^{\prime} \beta+\epsilon_{i} \quad$ Ecucación de interés(Deserción)

(2) $S_{i}^{*}=z_{i}^{\prime} \beta+\epsilon_{i} \quad$ Ecucación de selección(Acceso)

Luego la respuesta observada queda determinada por:

$y_{i h}=\left\{\begin{array}{l}y_{i 1} \text { (Des.defin)si }-\infty<y_{i}^{*} \leq k_{1} \wedge \text { si }=1 \\ y_{i 2} \text { (Des. temp)si } k_{1}<y_{i}^{*} \leq k_{2} \wedge \text { si }=1 \\ y_{i 3} \text { (Matr. cont)si } k_{2}<y_{i}^{*} \leq \infty+\wedge \text { si }=1\end{array}\right.$

Al estar condicionada la variable de interés por la ecuación de selección, los errores siguen una distribución conjunta normal bivariada.

Si la población que accede a la educación universitaria no es representativa de la población potencial (no cumple con una selección aleatoria), la correlación entre la ecuación de acceso y la de deserción será distinta de $0\left(p_{13} \quad 10\right)$. De ser así, la estimación de los coeficientes de la ecuación de deserción resultarán sesgados, si no se condiciona por el acceso a las carreras universitarias. Este modelo econométrico es bastante coherente con el modelo teórico propuesto por St.John et al. (2000), al modelar el proceso en etapas. Este modelo será llamado Selection Ordered Probit (SOP) de manera análoga a los modelos descritos por Miranda y Rabe-Hesketh (2005).

\section{El problema de la endogeneidad}

Uno de los problemas más comunes en la econometría es hacerse cargo de la endogeneidad. Esta puede ser causada por múltiples factores, como la omisión de variables relevantes, simultaneidad o multicausalidad, errores de medida o heterogeneidad individual no controlada, entre otros Roberts y Whited (2012).
Debido a que el término de error es no observable, no hay una forma empírica que pueda asegurar la existencia o no, de correlación entre el error y una variable explicativa (Roberts y Whited, 2012) $\mathrm{y}$, por ende, no se puede asegurar estadísticamente que el problema haya sido resuelto. Pese a ello, existen formas de disminuir el efecto de la endogeneidad. Un primer paso, es controlar con una gran cantidad de regresores, de manera de disminuir el sesgo de variable omitida (Jalan y Ravallion, 2003). Luego, existen distintos enfoques para disminuir el efecto en la endogeneidad de una variable en particular, como utilizar metodología de Variables Instrumentales, Matching o RD (Roberts y Whited, 2012).

Para estimar el acceso y la deserción universitaria, una de las variables de mayor interés a incluir en la especificación del modelo, son las ayudas estudiantiles. Es de suma relevancia analizar el efecto que estas tienen, ya que año a año el Estado invierte numerosos recursos en ellas (Comisión de Financiamiento Estudiantil para la Educación Superior, 2012). Sin embargo, muchos estudios develan los problemas de endogeneidad que conlleva el incluir esta variable (Cameron, 2009; Melguizo et al., 2011; Meneses y Blanco, 2010), principalmente, porque las ayudas estudiantiles no son asignadas de manera aleatoria, sino que en función de una serie de características del individuo, además de realizarse un proceso de postulación voluntario a ellas. Esto conlleva a que los individuos que se adjudican las ayudas estudiantiles, puedan ser también los individuos con mayor motivación o vulnerabilidad socioeconómica, lo cual no es posible de controlar del todo en la especificación. Finalmente, esto se traduce en que la variable que identifica si el individuo recibe una ayuda estudiantil, esté correlacionada con el término de error no observable, produciendo sesgos en los efectos reales de las ayudas estudiantiles sobre la variable de interés.

Para reducir la endogeneidad presente en las ayudas estudiantiles, se utiliza el enfoque de variable instrumental propuesto por (Meneses y Blanco, 2010). Este enfoque estima la probabilidad de adjudicarse una ayuda estudiantil por medio de una serie de variables explicativas y un instrumento que esté correlacionado con la variable a estimar, pero no con el error (Meneses \& Blanco, 2010).

Este modelo, utilizando una parametrización particular de los errores, es llamado Selection Endogenous Dummy Ordered Probit (SEDOP) (Bratti y Miranda, 2009).

Al estimar el modelo de deserción considerando el efecto de las demás ecuaciones (a diferencia de un modelo de ecuaciones totalmente independien- 
tes), el reducir el sesgo de variable omitida en una ecuación puede llevar a obtener estimaciones más precisas en la ecuación de interés, debido a la posible correlación entre los errores.

Con este modelo, se pretende reducir la endogeneidad presente en las ayudas estudiantiles en la ecuación de deserción y, de ser posible, en la ecuación de acceso. En particular, es posible distinguir 4 tipos de situaciones en relación a la posible endogeneidad de la variable explicativa (Bratti y Miranda, 2010):

El coeficiente de correlación $p_{13}\left(\right.$ o $\left.p_{23}\right)$ no es estadísticamente significativo y el coeficiente en la ecuación de Deserción (o Acceso) es estadísticamente significativo. En este caso, la variable que identifica la ayuda estudiantil (AE) es exógena con respecto a la ecuación de Deserción (o Acceso) y su efecto es causal.

El coeficiente de correlación $p_{13}\left(\mathrm{o} p_{23}\right)$ es estadísticamente significativo y el coeficiente de AE no es estadísticamente significativo. En este caso, la variable AE es endógena con respecto a la ecuación de Deserción (o Acceso) y la correlación presente entre la variable AE con la ecuación de Deserción (o Acceso) es producto de heterogeneidad no observable.

El coeficiente de correlación $p_{13}\left(\right.$ o $\left.p_{23}\right)$ es estadísticamente significativo y el coeficiente de AE también es estadísticamente significativo. En este caso, si bien la variable AE es endógena con respecto a la ecuación de Deserción (o Acceso), también posee un efecto causal en la ecuación de interés. Las estimaciones de ambos coeficientes darán una idea de la relativa importancia entre ambos efectos.

Tanto el coeficiente de correlación $p_{13}\left(\right.$ o $\left.p_{23}\right)$ como el coeficiente de AE, no son estadísticamente significativos. En este caso, no es posible determinar el efecto de la variable sobre la ecuación de interés.

\section{Implementación y estimación}

Para la implementación de los modelos ya descritos, se utiliza el comando cmp (Continuous Mixed Process Estimator) implementado en Stata 12 TM. Este permite estimar una gran familia de estimadores de multi-ecuaciones, multi-nivel y de procesos mixtos recursivos o condicionales (Roodman, 2009).

"Multi-ecuaciones", significa que cmp puede estimar sistemas SUR (Seemingly Unrelated) y de variables instrumentales. "Multi-nivel", significa que los efectos no observables pueden ser modelados a distintos niveles con relación jerárquica. "Pro- cesos mixtos", significa que cada ecuación puede tener una variable dependiente distinta, sin embargo, el término de error debe seguir una distribución Normal. "Recursivos", significa que cmp sólo puede estimar conjuntos de ecuaciones que poseen etapas claramente definidas, sin presentar una multicausalidad. Finalmente, "condicional", significa que el modelo a estimar puede variar por observación, es decir, una ecuación puede no ser considerada para aquellas observaciones en las cuales resulta irrelevante.

\section{Descripción de los datos}

Para analizar la deserción del sistema universitario, se considerará sólo a aquellos estudiantes que acceden a la universidad los años 2006 o 2007, habiendo rendido previamente el SIMCE en el año 2003 y para los cuales se cuente con información de la PSU 2006 o 2007. Además, se reducirá la muestra a las carreras de bachillerato, ciclo inicial o plan común; licenciatura no conducente a título y título profesional con licenciatura previa. Se eligió analizar sólo este subgrupo de carreras, debido a que son impartidas exclusivamente por universidades, dejando fuera a las carreras que también son impartidas por Centros de Formación Técnica o Institutos Profesionales.

Así, se obtiene una muestra de carreras más homogéneas, permitiendo un mejor ajuste de los modelos a estimar. Un aspecto clave, es que las carreras exclusivamente universitarias tienen una duración de al menos 8 semestres (considerando que los bachilleratos o ciclos iniciales o comunes conducen a una segunda carrera, tardando en total al menos 8 semestres en egresar). Al acotar la muestra a carreras que duran al menos 8 semestres es posible categorizar de buena forma el proceso de deserción del sistema universitario. Además, las carreras cuya duración es mayor a 4 años son clasificadas en el mismo nivel (nivel 6) (UNESCO, 2011).

De los 233.896 estudiantes para los cuales se cuenta con información relativa a la prueba SIMCE en el año 2003, sólo 125.470 rindieron la prueba PSU en el año 2005 o el año 2006 (proceso 2006 y proceso 2007). Este grupo de estudiantes es sobre el que se evaluará la decisión de acceder o no a una carrera universitaria (Muestra de Acceso). Finalmente, aquellos estudiantes de esta cohorte que accedieron en el año 2006 o el año 2007 a una carrera exclusivamente universitaria, conforman el grupo sobre el cual se evaluará la decisión de desertar o no del sistema universitario (Muestra de Deserción). Esta muestra corresponde a 58 de las 61 universidades a las cuales podían acceder los estudiantes 
en el año 2006, por lo que es una muestra bastante representativa del sistema universitario en su conjunto.

\section{Definición de deserción}

Para efectos del estudio se considerará como deserción general al proceso de abandono voluntario o forzoso del sistema universitario, de manera temporal o definitiva. Si bien el proceso forzoso puede deberse a causas distintas a las del proceso de carácter voluntario, el propósito de esta investigación es medir la probabilidad que un estudiante deserte del sistema universitario, dadas ciertas variables o características de entrada. En este sentido, no se están midiendo las causas directas de la deserción de los estudiantes, sino más bien la probabilidad de deserción del sistema universitario dadas ciertas características (del estudiante y externas a él) previas a la decisión de desertar (independiente de si ésta es de carácter voluntaria o forzosa). Dentro de la deserción general se diferenciará dos procesos: Deserción temporal y Deserción definitiva.

Como los estudiantes que acceden en el año 2007 sólo pueden ser analizados 5 años (ya que la información utilizada llega hasta el año 2011), la condición común para los dos grupos es que la Deserción definitiva puede ocurrir sólo el primero, segundo o tercer año desde el acceso al sistema universitario, verificándose que el estudiante no esté matriculado hasta el año 2011. En este sentido, la Deserción definitiva sería de al menos 2 años de ausencia para quienes acceden en el año 2007 y de al menos 3 años para quienes acceden en el año 2006.

Uno de los casos complejos de clasificar es el de los estudiantes que acceden en el año 2006 y permanecen 4 años seguidos, pero luego no están matriculados los años 2010 ni 2011. Este caso es clasificado como Matrícula continua, independiente de la duración de la carrera a la cual accede el estudiante inicialmente y de la realización previa de cambio de carrera o universidad ${ }^{1}$. Esta clasificación responde al hecho que se privilegia el cometer un error clasificando a un desertor como no desertor, que viceversa (en este caso límite).

Otro caso difícil de clasificar es el de Deserción temporal al quinto año. Debido a que las carreras universitarias pueden durar desde 8 semestres en adelante, aquellos estudiantes que permanecen 4 años seguidos en el sistema y luego desertan temporalmente (abandonan el quinto año y vuelven el

\footnotetext{
${ }^{1}$ Cuando se hable de la universidad o carrera del estudiante, se hace referencia a la carrera y universidad a la cual el estudiante ingresó inicialmente, ya sea en el año 2006 o en el año 2007.
}

sexto año) pueden haber egresado de su carrera, por lo que correspondería clasificarlos como Matrícula continua. Pese a ello, se optó por clasificar este caso como Deserción temporal, debido a la estadística descriptiva analizada.

Se puede apreciar que del total de los alumnos que acceden a una carrera universitaria el año 2006 y el año 2007, un $16 \%$ deserta definitivamente, un $12 \%$ deserta temporalmente y un $71 \%$ se mantiene continuamente en el sistema. De esta manera, se observa que la deserción general es de casi un $30 \%$.

En relación al cambio de carrera, más del $80 \%$ de aquellos que desertan definitivamente no se han cambiado de carrera previamente. Sin embargo, cerca del $70 \%$ de los estudiantes que desertan temporalmente, se cambia alguna vez de carrera (ya sea antes o después). Esto da luces que los estudiantes que desertan temporalmente, en su mayoría, no vuelven a su carrera de acceso.

Por otra parte, se observa que sólo el $8 \%$ de estudiantes que desertan definitivamente cambia de universidad de manera previa. Por otro lado, casi el $60 \%$ de los estudiantes que desertan temporalmente cambia de universidad, siendo altamente probable que el cambio sea posterior a la Deserción temporal. Finalmente, casi el $80 \%$ de los estudiantes que no desertan, se mantiene continuamente en la misma universidad.

Un $13 \%$ de estudiantes se cambia de universidad pero no de carrera (a una carrera con el mismo nombre ${ }^{2}$ ). Esto puede introducir un cierto ruido a los datos entregados anteriormente, ya que se había considerado que aquellos estudiantes que no se cambiaban de carrera (nombre) se mantenían en la misma institución. Por otro lado, para los efectos de categorizar la deserción no debería influir mayormente, ya que al cambiarse de institución a una misma carrera, el estudiante posiblemente podrá reconocer ciertas asignaturas ya cursadas y con ello no atrasarse.

En el caso de Deserción definitiva, el porcentaje real de cambio de carrera (carrera o institución) es cercano al $18 \%$. Para el caso de Deserción temporal, el cambio de carrera o institución es cercano al $80 \%$. Finalmente para aquellos estudiantes que no desertan, el cambio de carrera o institución es cercano al $52 \%$.

Finalmente, cerca de un $13 \%$ de estudiantes cam-

\footnotetext{
${ }^{2}$ Lamentablemente no se cuenta en la BBDD con un código que identifique únicamente a cada carrera, por lo que se utilizó el nombre de la carrera como identificador y la concatenación entre los nombre de carrera y universidad para la generación de ciertas variables, como el ranking al interior de la carrera.
} 
bia de universidad y no de carrera, implicando un cierto sesgo a la baja en las tasas de cambio de carrera expuestas anteriormente. Sin embargo, para el caso de Deserción definitiva, la mayoría de los estudiantes no experimentan ni cambios de universidad ni de carrera previamente, evidenciándose un cambio de carrera menor al $20 \%$ y sólo un $8 \%$ cambia de universidad previamente a la deserción definitiva. Para el caso de Deserción temporal, cerca del $80 \%$ de los estudiantes experimenta un cambio de universidad o carrera, y el $50 \%$ cambia de universidad y de carrera. Esto da cuenta que el fenómeno de deserción temporal está íntimamente ligado con el de cambio de carrera.

\section{Agrupación áreas CINE UNESCO}

Para poder analizar posibles diferencias entre las distintas áreas de carrera a la cual accede el estudiante, se optó por agruparlas en categorías homogéneas, de manera de facilitar la interpretación de los resultados, sin perder la heterogeneidad presente en las distintas áreas.

Para categorizar los distintos tipos de carrera, se tomó como marco la adaptación chilena a la Clasificación Internacional Normalizada de la Educación (CINE) (UNESCO, 2011) y la agrupación por área de conocimiento utilizada por Humlum et al. (2007). Teniendo como criterio mantener grupos relativamente homogéneos, en términos de tasas de deserción, áreas de conocimiento y masa, se agruparon de la siguiente manera: a) Salud: Salud; b) Educación y Humanidades: Educación, Humanidades; c) Ciencias: Tecnología, Ciencias Exactas y Naturales, Agropecuaria; y d) Ciencias Sociales: Ciencias Sociales, Derecho, Administración y Comercio, Arte y Arquitectura.

La muestra seleccionada incluye a 58 de las 61 universidades a las cuales los estudiantes podían acceder en los años 2006 o 2007. Para clasificar a las universidades según su selectividad, se utilizó la clasificación propuesta por Mizala, Makovec y Hernández en el FONIDE N F511059 (2011): a) Universidades CRUCH con liderazgo académico, b) Universidades CRUCH con baja selectividad, c) Universidades privadas no tradicionales selectivas, y d) Universidades privadas no tradicionales con baja selectividad.

\section{Variables}

\section{Ecuación de deserción}

La habilidad individual del estudiante, es aproximada por el quintil del alumno en la distribución nacional de la PSU individual de matemática, por el quintil del alumno en la distribución nacional de la PSU individual de lenguaje, por el quintil del NEM del alumno y por el ranking del alumno en el colegio donde rindió el SIMCE (también medido como quintil). La inclusión de estas variables como quintil permite reflejar efectos no lineales en ellas, como lo exponen Acuña et al. (2010). Para poder controlar también por la habilidad relativa del estudiante en la carrera a la cual accede inicialmente, se incluyó además el quintil de puntaje promedio PSU lenguaje matemática, previamente normalizado en la carrera.

Cabe notar que los intervalos de puntaje en cada quintil pueden traslaparse. Esto es debido a que las variables que identifican los quintiles, fueron construidas en relación a la población de la base de datos que rindió la prueba PSU en el año 2005 (proceso 2006), o en relación a la población de la base de datos que rindió la prueba PSU en el año 2006 (proceso 2007), por lo que no hay una relación absoluta con el puntaje obtenido, sino relativa a la población que rindió la PSU en dicho año (considerando sólo a quienes rindieron el SIMCE en el año 2003).

Las características del establecimiento educacional al que asistió el estudiante, son medidas por el quintil en la distribución nacional de la PSU de matemática a nivel de establecimientos, el tipo de colegio (científico-humanista vs. técnico profesional), la dependencia del colegio (particular pagado, municipal o particular subvencionado), el índice de vulnerabilidad escolar (IVE) asociado al establecimiento educacional, si éste es un liceo emblemático, si es religioso y si es un establecimiento mixto (respecto a sólo de hombres o mujeres). Todas estas variables, son reportadas en la encuesta de la PSU que cada estudiante rindió, correspondiendo por ende, a características del establecimiento en el cual el estudiante rindió cuarto medio (salvo la variable "liceo emblemático" que se construye a partir del RBD del establecimiento).

Las características demográficas y socioeconómicas del hogar de pertenencia del alumno, considera las siguientes variables explicativas: ingreso mensual del hogar reportado en la base de datos del SIMCE (categorizado para capturar posibles efectos no lineales), el nivel educacional del padre y la madre, si el jefe del hogar es el padre del alumno, si los padres del alumno proveerán el financiamien- 
to principal de su carrera universitaria, el número de hermanos menores del alumno que cursan educación básica, el género del alumno, si el alumno proviene de una región distinta a la de la carrera, si el alumno accede en el año 2006 a la universidad (con respecto a acceder en el año 2007) y la edad del alumno previamente categorizada.

Se analiza, también, la proporción de estudiantes que recibe una ayuda estudiantil, pese a que esta variable se incluye como una dummy aparte en el modelo. Para poder corregir la endogeneidad presente en las ayudas estudiantiles, se optó por considerar en una sola dummy si el estudiante percibe o no una ayuda estudiantil (juntando las categorías "Sólo crédito", "Beca y crédito" y "Sólo beca"), resultando también categorías más balanceadas.

Las características de la institución de Educación Superior y de la carrera a la cual accede el estudiante son medidas por las siguientes variables explicativas: el tipo de universidad, la macroárea de conocimiento a la que pertenece la carrera, el arancel de carrera categorizado, los años de acreditación de la universidad (con respecto al no estar acreditada $)^{3}$, el promedio PSU que reporta la universidad respecto a sus estudiantes matriculados en $2010^{4}$ y el número de JCE de Doctorado de la universidad $^{5}$. Se incluyen también características del mercado laboral de la carrera, considerando solo la variable que identifica la probabilidad de obtener empleo al segundo año de egreso.

\section{Ecuación de acceso}

La habilidad individual del estudiante es aproximada por el quintil del alumno en la distribución nacional de la PSU individual de matemática, por el quintil del alumno en la distribución nacional de la PSU individual de lenguaje, por el quintil del NEM del alumno y por el ranking del alumno en el colegio donde rindió el SIMCE (también medido como quintil). Siguiendo la especificación utilizada por Meneses y Blanco (2010), se incluye además el quintil en la distribución nacional del SIMCE de matemática ${ }^{6}$.

Las características del establecimiento educacional al que asistió el estudiante, para la ecuación de acceso, son medidas por el quintil en la distribución nacional de la PSU matemática a nivel de estable-

\footnotetext{
${ }^{3}$ Información obtenida de SIES, Años de Acreditación (14 de diciembre de 2010).

${ }^{4}$ Promedio PSU (lenguaje y matemática) de estudiantes de 1 er año 2010.

${ }^{5}$ Promedio PSU (lenguaje y matemática) de estudiantes de 1 er año 2010.

${ }^{6}$ No se incluyó la variable equivalente de lenguaje para mejorar la convergencia del modelo.
}

cimientos, el quintil en la distribución nacional del SIMCE de matemática a nivel de establecimientos, el tipo de colegio (científico-humanista vs. técnico profesional), la dependencia del colegio (particular pagado, municipal o particular subvencionado), el índice de vulnerabilidad escolar (IVE) asociado al establecimiento educacional, si éste es un liceo emblemático, si es religioso y si es un establecimiento mixto (respecto a sólo de hombres o mujeres). Todas estas variables son reportadas en la encuesta de la PSU que cada estudiante rindió, correspondiendo, por ende, a características del establecimiento en el cual el estudiante rindió cuarto medio.

Las características demográficas y socioeconómicas del hogar de pertenencia del alumno, para la ecuación de acceso, considera las siguientes variables explicativas: ingreso mensual del hogar reportado en la base de datos del SIMCE (categorizado para capturar posibles efectos no lineales), el nivel educacional del padre y la madre, si el jefe del hogar es el padre del alumno, si los padres del alumno proveerán el financiamiento principal de su carrera universitaria, el número de hermanos menores del alumno que cursan educación básica y el género del alumno ${ }^{7}$.

\section{Ecuación de ayuda estudiantil}

La habilidad individual de los alumnos es medida por medio del puntaje promedio lenguaje matemática que obtuvo el estudiante en la prueba SIMCE, el puntaje promedio lenguaje matemática que obtuvo el estudiante en la prueba PSU, el ranking del estudiante en el establecimiento de origen y el puntaje NEM. La inclusión de ciertas variables como puntajes promedios o sin considerar la distribución en quintiles, es debido a que se está emulando la especificación utilizada por Meneses y Blanco (2010), tratando de enriquecerla con aquellas variables que resulten pertinentes.

Cabe notar, que si bien en otro set de variables se incluyen dummies relacionadas con el puntaje de PSU promedio que obtuvo el estudiante, ellas pretenden capturar las condiciones de adjudicación de las ayudas estudiantiles (ambos son criterios de adjudicación para becas y créditos).

Las características demográficas y socioeconómicas del hogar de pertenencia del alumno, para la ecuación de Ayuda Estudiantil, son las mismas que las consideradas para la ecuación de Acceso.

Las características del establecimiento educa-

\footnotetext{
${ }^{7}$ Algunas variables demográficas y socioeconómicas de interés fueron excluidas, para mejorar la convergencia del modelo.
} 
cional al que asistió el estudiante, para la ecuación de Ayuda Estudiantil, son el tipo de colegio (científico-humanista vs. técnico profesional), la dependencia del colegio (particular pagado, municipal o particular subvencionado), el índice de vulnerabilidad escolar (IVE) asociado al establecimiento educacional, si éste es un liceo emblemático, si es religioso y si es un establecimiento mixto.

Las variables correlacionadas con los criterios de selección de las ayudas estudiantiles, son similares a las propuestas por Meneses y Blanco (2010). Específicamente, las variables explicativas consideradas son: una dummy que identifica si el estudiante posee un puntaje PSU superior a 550 ptos. (promedio entre lenguaje y matemática) o no y una dummy similar a la anterior pero con un puntaje de corte de 500 ptos. Cabe notar, que si bien el nivel de ingreso del grupo familiar, es una variable considerada en los criterios de selección, esta ya está incluida dentro de las características demográficas y socioeconómicas.

Al igual que con el set anterior de variables, las variables de exclusión son similares a las propuestas por Meneses y Blanco (2010). Debido a que en la base de datos no se contaba con la información necesaria para construir las variables de exclusión originales, se construyó proxys de las mismas. Las variables utilizadas son la proporción de estudiantes del establecimiento de origen (aproximando la proporción de repitentes del establecimiento de origen) que rinde la PSU en el año 2005 y la proporción de estudiantes del establecimiento de origen (aproximando la proporción de estudiantes que postula a una ayuda estudiantil el año anterior) que postula al CAE para el año 2005. Este instrumento está claramente correlacionado con la probabilidad de obtener una ayuda estudiantil y no es parte de un proceso de maximización individual de utilidades, por lo que es poco probable que se correlacione con el error. Por ello, estas variables de exclusión deberían corregir en parte la endogeneidad de las ayudas estudiantiles, disminuyendo el sesgo en los coeficientes estimados.

\section{Resultados}

\section{Modelo de tres ecucaciones}

La estimación del modelo SEDOP dio como resultado:

$$
\begin{aligned}
& p_{12}=-, 0325188 \\
& p_{13}=-, 0897411 \\
& p_{23}=-, 5336991
\end{aligned}
$$

Los coeficientes de correlación $p_{13}$ y $p_{23}$ son estadísticamente significativos al $95 \%$ de confianza, lo cual puede apreciarse en que los intervalos de confianza de estos parámetros excluyen el $0^{8}$.

$$
\begin{array}{r}
p_{12} \in[-, 0935125 ;, 0287182] \\
p_{13} \in[-, 1406317 ;-, 0383775] \\
p_{23} \in[-, 5679086 ;-, 4976489]
\end{array}
$$

Esto implica que existe una correlación significativa entre los errores de la ecuación de Acceso y la de Ayuda Estudiantil, y también una correlación significativa entre la ecuación de Deserción y la de Ayuda Estudiantil.

Por otro lado, el coeficiente $p_{12}$ resulta no significativo, por lo que la corrección por la ecuación de Acceso no influye directamente en los coeficientes estimados en la ecuación de Deserción. Esto puede deberse a que, al controlar con una gran cantidad de regresores, se logró eliminar el sesgo directo producido por la selección de muestra. Sin embargo, pese a que la corrección por Acceso no influya de manera directa, sí lo hace de manera indirecta al correlacionarse con la estimación de la variable que identifica el recibir una ayuda estudiantil (AE), por lo que excluir esta ecuación del modelo podría aumentar el sesgo en la ecuación de Deserción ${ }^{9}$.

Si bien los coeficientes de correlación $p_{13}$ y $p_{23}$ son estadísticamente significativos, el coeficiente de AE también es estadísticamente significativo en ambas ecuaciones (Acceso y Deserción). En este caso, la variable $\mathrm{AE}$ es endógena con respecto a las ecuaciones de Acceso y Deserción, pero también posee un efecto causal en ambas ecuaciones. Las estimaciones de ambos coeficientes entregan una idea de la relativa importancia entre ambos efectos. Analizando los coeficientes estimados, puede notarse que en el caso del coeficiente que identifica las ayudas estudiantiles en la ecuación de Deserción, el peso de la variable es de una gran magnitud en relación a las demás $(0,4)$ y el coeficiente de correlación es cercano a $|0,09|$, por lo que puede inferirse que el

\footnotetext{
${ }^{8} \mathrm{El}$ comando cmp no entrega un valor de $\mathrm{p}$-value que dé cuenta de la significatividad del parámetro p, sin embargo, sí entrega los intervalos de confianza al $95 \%$ de confianza.

${ }^{9} \mathrm{Al}$ cambiar la población potencial que accede al sistema universitario, por todos los estudiantes que rindieron el SIMCE en el año 2003, el coeficiente de correlación $p_{12}$ también resultó no significativo.
} 
efecto de la endogeneidad es relativamente menor al de la causalidad. Es decir, la obtención de una ayuda estudiantil explica de mejor manera el valor del coeficiente obtenido, que la heterogeneidad individual producto de algún error de medición o de variable omitida. Este resultado sustenta a su vez, que la especificación elegida para modelar la ecuación de deserción es suficientemente robusta, ya que logra disminuir en gran parte la endogeneidad producida por el sesgo de selección en el acceso y por la obtención de una ayuda estudiantil.

Otro aspecto a notar, es que el signo de los coeficientes de correlación es negativo en ambos casos, lo que indicaría que los efectos de la heterogeneidad individual en la probabilidad de obtención de las ayudas estudiantiles, afectan negativamente la heterogeneidad individual, en la probabilidad de acceso a la universidad y en la probabilidad de no desertar definitivamente (recordando que la opción de mayor utilidad latente en la ecuación de Deserción la proporciona la categoría de Matrícula continua).

Una posible explicación de esta correlación negativa, entre la probabilidad de obtener una ayuda estudiantil y la de acceder a la universidad sin desertar posteriormente, es que la población que postula a las ayudas estudiantiles es más vulnerable y el nivel de ingreso familiar no logra capturar por completo esa vulnerabilidad socioeconómica. En este sentido, la heterogeneidad no observable en la probabilidad de recibir una ayuda estudiantil (vulnerabilidad, entre otros factores) estaría correlacionada negativamente con la probabilidad de acceder a la universidad y no desertar posteriormente.

A continuación se presentan los efectos marginales, comparando el modelo condicionado por acceso y corregido por la endogeneidad de las ayudas estudiantiles (SEDOP), con el modelo sin condicionar por acceso ni corregir la endogeneidad de las ayudas estudiantiles (OP). Se analizan gráficamente las variables de mayor interés en cada set de variables.

\section{Habilidad Individual}

Con respecto a la habilidad medida por el puntaje individual por quintil en PSU matemática y lenguaje, se aprecian efectos estadísticamente significativos en los extremos de la distribución. En el caso de la prueba de matemática, los estudiantes que pertenecen al quintil de mayor puntaje ("V"), poseen un $30 \%$ menos de probabilidad de desertar definitivamente que aquellos pertenecientes al tercer quintil. Por otro lado, en el caso de la prueba de lenguaje, el efecto resulta estadísticamente significativo y de magnitud considerable sólo para los estudiantes pertenecientes al quintil de puntajes más bajos. Los estudiantes pertenecientes al quintil de menores puntajes en la prueba PSU de lenguaje, presentan cerca de un $20 \%$ más de probabilidad de desertar definitivamente que aquellos pertenecientes al tercer quintil.

Con respecto al quintil del estudiante según el NEM, se aprecian efectos de mayor magnitud comparados a los de la PSU matemática y lenguaje. Los estudiantes pertenecientes al quintil de mayor puntaje NEM ("V"), presentan cerca de un $50 \%$ menos de probabilidad de desertar definitivamente, que aquellos pertenecientes al tercer quintil. Por otro lado, aquellos estudiantes que pertenecen al quintil de menor puntaje NEM ( "I") poseen entre un $30 \%$ y un $40 \%$ más de probabilidad de desertar definitivamente que aquellos estudiantes pertenecientes al tercer quintil de NEM.

$\mathrm{Al}$ analizar el ranking de notas del estudiante en su establecimiento de origen, se aprecian diferencias estadísticamente significativas aun habiendo controlado por el NEM del estudiante. Los estudiantes pertenecientes al quintil de mayor ranking de notas en su establecimiento de origen ("V"), poseen entre un $30 \%$ y un $40 \%$ menos de probabilidad de desertar definitivamente que aquellos pertenecientes al tercer quintil. Por otro lado, los estudiantes pertenecientes al quintil de menor ranking ("I") presentan entre un $30 \%$ y un $40 \%$ más de probabilidad de desertar definitivamente, al comparar con la categoría de referencia. Al comparar los modelos SEDOP (Controlando) y OP (Sin Controlar), no se aprecian diferencias sustantivas.

Con respecto al ranking que posee el estudiante en su carrera (según el puntaje promedio PSU lenguaje-matemática normalizado), puede observarse que el efecto sobre la deserción universitaria es estadísticamente significativo solamente para los primeros 2 quintiles, siendo de una magnitud relevante solamente para el quintil de menor ranking de PSU dentro de la carrera. Los estudiantes pertenecientes al quintil de menor ranking ("I") poseen cerca de un $30 \%$ más de probabilidad de desertar definitivamente que los pertenecientes al tercer quintil. Esto indicaría que la posición del estudiante dentro de la carrera es un factor relevante sobre la deserción universitaria, solamente si el estudiante está en el extremo inferior del ranking, teniendo una mayor probabilidad de deserción aquellos estudiantes que están peor ranqueados en la carrera a la cual acceden.

\section{Calidad Establecimiento de Origen}

Con respecto al quintil de PSU matemática del establecimiento de origen, se aprecia que es un fac- 
tor de mayor poder explicativo que los quintiles de PSU matemática y lenguaje individual, llegando los estudiantes que pertenecen al quinto quintil a tener cerca de un $40 \%$ menos de probabilidad de desertar definitivamente, que aquellos pertenecientes a establecimientos en el tercer quintil. Por otro lado, los estudiantes pertenecientes a establecimientos en el quintil de menores puntajes en la PSU matemática (满”), poseen un $40 \%$ más de probabilidad de desertar definitivamente que aquellos pertenecientes a establecimientos en el tercer quintil.

Cabe notar que a diferencia del quintil individual, esta es una medida agregada a nivel del establecimiento, por lo que captura en cierta medida la calidad del establecimiento de origen del estudiante. El encontrar un efecto mayor del quintil del establecimiento de origen al individual, implica que el efecto de provenir de un buen establecimiento, sobre la deserción universitaria, es mayor que el efecto de la habilidad individual medida por la PSU individual de lenguaje y matemática. No se aprecian mayores diferencias entre la estimación del modelo SEDOP con el OP para esta variable en cuestión.

Al analizar el grado de vulnerabilidad del establecimiento de origen puede apreciarse que los estudiantes que provienen de establecimientos del quintil de mayor índice de vulnerabilidad económica ( "V"), poseen cerca de un $30 \%$ más de probabilidad de desertar definitivamente, al comparar con los establecimientos de menor vulnerabilidad económica ("No tienen IVE"). Este efecto es claramente no lineal, siendo estadísticamente no significativa la comparación con los primeros dos quintiles de IVE y la categoría de referencia. En este sentido, la vulnerabilidad económica del establecimiento de origen afectaría la deserción universitaria sólo desde cierto nivel de vulnerabilidad.

Con respecto a otras variables que identifican las características del establecimiento de origen del estudiante, se aprecia que: la probabilidad de desertar definitivamente de los estudiantes de establecimientos particulares pagados es un $13 \%$ menor que los pertenecientes a establecimientos municipales; los científico-humanista un $17 \%$ mayor que los técnico-profesionales; los colegios exclusivamente de mujeres un $9 \%$ menor que los mixtos; los emblemáticos un $9 \%$ menor que los no emblemáticos; y los religiosos cerca de un $20 \%$ menor que los no religiosos. Todos estos efectos son estadísticamente significativos al $95 \%$ de confianza y dan cuenta de la influencia que tiene la calidad y el tipo de establecimiento del cual proviene el estudiante, sobre la deserción universitaria.

\section{Características Socioeconómicas y De- mográficas}

Con respecto al nivel de ingreso familiar, proporcionado por la encuesta del SIMCE, puede apreciarse un claro efecto sobre la deserción, disminuyendo la probabilidad de desertar definitiva y temporalmente a medida que aumenta el nivel de ingreso. Los estudiantes pertenecientes al tramo de mayor ingreso $(>1,800)$ poseen cerca de un $60 \%$ menos de probabilidad de desertar definitivamente comparando con la categoría de referencia $(<200)$.

Cabe notar que con distintas especificaciones se exhibían algunas diferencias entre los modelos SEDOP y OP (especialmente al incluir pocas variables en las ecuaciones), aumentando el efecto del ingreso sobre la deserción una vez que se corregía por acceso y por la endogeneidad de las ayudas estudiantiles. Esto se debe principalmente a la corrección de la endogeneidad presente en las ayudas estudiantiles, ya que su obtención está altamente correlacionada al nivel de ingreso familiar y a los resultados académicos del estudiante (presente en los criterios de asignación). Sin embargo, esta diferencia se atenuó al incluir más variables en el modelo, volviendo la especificación más robusta.

Con respecto al nivel educacional de los padres, puede observarse que sólo el nivel educacional de la madre es significativo para explicar la deserción universitaria. La variable tiene mayor poder explicativo para la categoría "Profesional", indicando que aquellos estudiantes cuyas madres tienen estudios de nivel Profesional (al contestar la encuesta del SIMCE en el año 2003) poseen una probabilidad estadísticamente significativa menor de desertar definitivamente que aquellos estudiantes cuyas madres sólo poseen educación básica, ya sea completa o incompleta. Claramente el efecto de esta variable es bastante menor al del ingreso o la habilidad individual y la calidad del establecimiento de origen del estudiante.

Las ayudas estudiantiles se posicionan como uno de los factores de mayor relevancia en la disminución de la deserción universitaria. Un estudiante que posee beca, crédito, o ambos beneficios, presenta una probabilidad un $70 \%$ menor de desertar definitivamente, que un estudiante que no percibe ayuda estudiantil alguna. Esta variable aumenta considerablemente su efecto al corregir por la endogeneidad de las ayudas estudiantiles y por el acceso a las carreras universitarias. La diferencia de probabilidad de desertar definitivamente entre los estudiantes que perciben o no una ayuda estudiantil, varía de cerca de un $40 \%$ a un $70 \%$ al comparar los dos modelos estimados. 
Cabe notar que esta variable fue estimada con un enfoque de variable instrumental, sustituyéndose la variable de "obtención de alguna ayuda estudiantil" por la "probabilidad de obtener una ayuda estudiantil", lo que permite corregir el sesgo de la estimación sobre la probabilidad de deserción universitaria presente en esta variable. Si la endogeneidad fue corregida con éxito (dependiendo de la fortaleza del instrumento utilizado), se podría concluir que las ayudas estudiantiles poseen una relación de causalidad positiva con la disminución de la deserción (tanto temporal como definitiva) y no sólo una correlación estadística.

Con respecto a otras variables que identifican las características socioeconómicas y demográficas del estudiante y su núcleo familiar, se aprecia que: la probabilidad de desertar definitivamente de las mujeres es un $15 \%$ menor que la de los hombres; si el padre del alumno es el jefe del hogar un $15 \%$ menos que si no lo es; si los padres son financiadores principales un $6 \%$ menor que si no lo son; si el estudiante tiene dos o más hermanos en básica o prebásica, un $29 \%$ mayor que si no tiene; si el estudiante accede en el año 2006 a la universidad, un $16 \%$ mayor que si accede en el año 2007; si el estudiante es mayor de 18 años, un $14 \%$ mayor que si el estudiante tiene 18 años al ingresar a la universidad; y si el estudiante proviene de región con respecto a la carrera a la cual accede, un $6 \%$ mayor que si proviene de la misma región de la carrera. Todos estos efectos son estadísticamente significativos al $95 \%$ de confianza y dan cuenta de la influencia que tienen las características socioeconómicas y demográficas del estudiante y su núcleo familiar, sobre la deserción universitaria.

\section{Características de la Universidad y Carrera}

Al analizar las variables que identifican las características de la universidad a la que accede el estudiante, hay que tener en cuenta que la interpretación debe realizarse de manera conjunta, ya que las variables están altamente correlacionadas y distintas categorías identifican conjuntos similares de universidades. Por ejemplo, las universidades que poseen un promedio de PSU menor a 500 puntos son todas universidades no tradicionales de baja selectividad y, por otro lado, no existen universidades de este grupo que posean un promedio PSU entre 600 y 700 puntos. Pese a ello no existe multicolinealidad entre estas variables y se mantiene cierta varianza, por lo que la estimación se efectúa sin problemas.

Al analizar el tipo de universidad según su selectividad, se aprecia que sólo resulta estadísticamente significativa la comparación entre la categoría "Pri- vadas No Selectivas" y la categoría de referencia ("Privadas Selectivas"). Los estudiantes que acceden a universidades de la categoría "Privadas No Selectivas" presentan un $20 \%$ menos de probabilidad de desertar definitivamente, que aquellos estudiantes que acceden a universidades de la categoría de referencia ("Privadas") Selectivas

Con respecto a los años de acreditación de la universidad, los estudiantes que acceden a universidades acreditadas "Entre 6 y 7 años" tienen una probabilidad de desertar definitivamente un $30 \%$ menor, que aquellos estudiantes que acceden a universidades de la categoría "No Acreditadas". Por otro lado se observa que no existe diferencias sustanciales en el efecto sobre la deserción entre cada categoría de acreditación. La diferencia, en cambio, se da al comparar con la categoría de referencia (universidades "No Acreditadas"), por lo que se infiere que no existe diferencias significativas entre los años de acreditación en su respuesta frente a la deserción, sino más bien, si la universidad está o no acreditada.

Los estudiantes que acceden a casas de estudio con un promedio PSU (de la universidad) mayor a 500 puntos ("[500-600]" y "[600-700]"), poseen una probabilidad $60 \%$ menor de desertar definitivamente que aquellos estudiantes que acceden a universidades con un promedio PSU menor a 500 puntos. No se aprecian diferencias sustantivas entre el efecto de pertenecer a la categoría de "[500-600]" o a la categoría de "[600-700]". Esta variable institucional en particular es la que presenta un mayor efecto sobre la deserción universitaria.

Finalmente, en relación con las características de la universidad a la cual accede el estudiante, se puede apreciar que el tipo de universidad no resulta una variable sustancial para determinar la deserción universitaria (en función de su significatividad estadística y magnitud del efecto) y que el efecto de la calidad de la institución es capturado de mejor manera por los años de acreditación y, sobre todo, por el promedio de PSU de la universidad. En este sentido se podría concluir que el acceder a una universidad de mejor calidad afecta negativamente la probabilidad de deserción de los estudiantes (disminuye la probabilidad de deserción), o más bien, el acceder a universidades de muy baja calidad (no acreditadas y con un puntaje PSU menor a 500 puntos) afecta positiva y sustancialmente la probabilidad de deserción de los estudiantes del sistema universitario (aumenta la probabilidad de deserción).

Cabe notar que el efecto calculado es el efecto individual de la variable sobre la probabilidad de deserción universitaria, pero pueden existir efectos 
de interacción con la variable que identifica el tipo de universidad según su selectividad, los cuales sí sean estadísticamente significativos al $95 \%$ de confianza.

Con respecto a la macroárea de conocimiento a la que pertenece la carrera del estudiante, se observa que existen efectos estadísticamente significativos y sustanciales para la categoría "Educación y Humanidades" y para "Ciencias", al comparar con la categoría de referencia ("Ciencias Sociales"). Cabe notar que aquellos estudiantes que acceden a una carrera de la categoría "Educación y Humanidades" (el efecto es probablemente debido a las carreras de pedagogía, debido a que concentra la mayor masa dentro de la categoría) poseen un $40 \%$ menos de probabilidad de desertar definitivamente, que aquellos que acceden a carreras de "Ciencias Sociales". Por otro lado, aquellos estudiantes que acceden a carreras de la macroárea de "Ciencias" poseen cerca de un $20 \%$ más de probabilidad de desertar definitivamente, que aquellos estudiantes que acceden a carreras de "Ciencias Sociales".

Este resultado es muy interesante, debido a que la estadística descriptiva mostraba que las mayores tasas de deserción se concentraban en carreras de la macroárea de "Educación y Humanidades", pero el efecto marginal de acceder a una carrera de la macroárea de "Educación y Humanidades" es negativo con respecto a la categoría de referencia. Esto puede deberse a que los estudiantes que acceden a carreras de la macroárea de "Educación y Humanidades" poseen intrínsecamente una mayor probabilidad de desertar (ya sea por menor habilidad individual, menor calidad del establecimiento de origen, menor ingreso familiar, etc.), lo que determina las altas tasas de deserción en esa macroárea. Esta diferencia, en términos estadísticos, se produce porque en la estimación del modelo econométrico se está controlando por más variables que sólo la macroárea de conocimiento, logrando capturar el efecto marginal.

Por otra parte, las carreras de la macroárea de "Ciencias" exhiben una probabilidad mayor de desertar definitivamente que las carreras de "Ciencias Sociales", pese a que poseen una menor tasa de Deserción definitiva. Una posible explicación a este resultado es que las carreras de la macroárea de "Ciencias", puedan tener un mayor nivel de exigencia académica, por lo que marginalmente afectarían al estudiante aumentando la probabilidad de deserción con respecto a "Ciencias Sociales". Pese a ello los estudiantes que acceden a estas carreras pueden tener una menor probabilidad intrínseca de desertar, lo que compensaría, en parte, el efecto de la macroárea sobre la deserción universitaria, en comparación a carreras de "Educación y Humanidades"

\section{o "Ciencias Sociales".}

En el caso de la macroárea de "Salud" el efecto respecto a la categoría de referencia es similar al de la macroárea de "Educación y Humanidades", pero más atenuado. Los estudiantes que acceden a carreras de la macroarea de "Salud" poseen cerca de un $10 \%$ menos de probabilidad de desertar definitivamente, al comparar con la categoría de referencia. Este efecto concuerda con la estadística descriptiva, ya que las carreras de la macroárea de "Salud" presentan menores tasas de Deserción definitiva que la macroárea de "Ciencias Sociales". Es posible que variables no medidas como la vocación influyan de manera más determinante en la deserción universitaria, lo que se podría estar reflejando en los efectos negativos capturados por las macroáreas de "Educación y Humanidades" y "Salud", respecto a la categoría de referencia.

El arancel de carrera no es un factor significativo y sustancial sobre la deserción universitaria. Esto puede deberse a que la restricción presupuestaria ya está siendo identificada por las variables de ingreso familiar y por el poseer o no una ayuda estudiantil. En este sentido, lo relevante no es qué tan elevado es el arancel de la carrera sino, más bien, si el estudiante en cuestión puede costearla de alguna forma (mayor ingreso familiar o con ayudas estudiantiles).

En relación con la realidad del mercado laboral para la carrera, se consideraron dos variables: ingreso futuro de la carrera al cuarto año de egreso y la probabilidad de empleo de la carrera al segundo año de egreso. Ambas variables están agregadas por cada carrera sin diferenciarse a nivel de universidades, por lo que no reflejan de buena forma la expectativa que posee cada estudiante sobre su futuro laboral al egresar. En el caso de la empleabilidad el ruido de este efecto es atenuado, debido a que independiente de la universidad de la cual el estudiante egrese, existe una menor varianza que en el caso del ingreso futuro. Esto es debido a que la variable es construida como una razón sobre el total de personas de esa carrera que buscan empleo. En otras palabras, si la empleabilidad de una carrera es cercana al $100 \%$, esta medida será similar dentro de la carrera independiente de la universidad de la cual se egrese, pero no así su ingreso esperado. Por ello se decidió controlar solamente por la probabilidad de empleo ${ }^{10}$ y dejar fuera la variable que identificaba el ingreso futuro de la carrera.

El efecto de la probabilidad de empleo es estadísticamente significativo y bastante relevante so-

\footnotetext{
${ }^{10} \mathrm{Al}$ incluir la variable de probabilidad de empleo, se disminuía la cantidad de observaciones de 52.000 a 44.000 , por lo que se generó la categoría "Sin Información" para no perder observaciones.
} 
bre la probabilidad de deserción del sistema universitario. Puede apreciarse que aquellos estudiantes que acceden a carreras con una empleabilidad al segundo año entre el $80 \%$ y el $90 \%$, poseen una probabilidad un $30 \%$ menor de desertar definitivamente, que aquellos estudiantes que acceden a carreras con una probabilidad de empleo menor al $80 \%$ al segundo año de egreso (categoría de referencia). Este efecto se atenúa al comparar con las carreras que presentan una probabilidad de empleo entre el $90 \%$ y el $100 \%$, pero sigue resultando estadísticamente significativo al $95 \%$ de confianza.

Una posible explicación de este resultado es que los estudiantes de carreras con mejor empleabilidad tendrían menos incentivos a desertar temporal o definitivamente que aquellos que acceden a carreras de baja empleabilidad, porque la rentabilidad esperada es mayor si se compara carreras con ingresos futuros similares.

Pese a los resultados obtenidos es necesario tener mejor información para hacer un análisis más certero, ya que no se cuenta con datos para esta variable en casi el $10 \%$ de la muestra final.

\section{Modelo por tipo de carrera y ma- croárea}

Para contrastar si existen efectos heterogéneos, de los factores determinantes de la deserción por tipo de universidad y macroárea de conocimiento, se realizó inicialmente una estimación de modelos separados para cada tipo de universidad y macroárea. A partir de los resultados obtenidos por cada modelo se pudo apreciar que existen efectos heterogéneos para algunas variables. Las variables identificadas fueron el quintil de PSU matemática individual, el quintil de PSU matemática del establecimiento de origen, el quintil de NEM individual, el ranking de notas del estudiante en el establecimiento de origen, el nivel de ingreso socioeconómico del núcleo familiar, el poseer una ayuda estudiantil y la acreditación de la universidad a la cual accede el estudiante.

Pese a notarse gráficamente las diferencias en los resultados entre cada modelo, no es posible realizar un test estadístico que permita comprobar si las diferencias en los factores es estadísticamente significativa, debido a que son modelos estimados sobre muestras distintas. Para testear estadísticamente si las diferencias son significativas, se estimó el modelo general OP ${ }^{11}$ incluyendo variables de interacción.

\footnotetext{
${ }^{11}$ Se optó por el modelo sin corrección endógena, debido a la gran cantidad de variables que involucraba el modelo SEDOP en este caso, dificultándose la convergencia.
}

De las variables de interés ya mencionadas, se excluyó de la interacción a la variable que identifica si el estudiante recibe una ayuda estudiantil o no, debido a que no pudo corregirse su endogeneidad al incluir tantas variables en la ecuación de interés.

\section{Efectos de la selectividad de la universidad}

No se aprecian efectos claros para la interacción entre el tipo de universidad según su selectividad y el quintil de PSU matemática individual del estudiante, el quintil de PSU lenguaje individual del estudiante, el quintil de puntaje NEM del estudiante ni para la interacción con el quintil de PSU matemática del establecimiento de origen del estudiante.

Cabe notar que el efecto marginal calculado es el efecto promedio del "tipo de universidad según su selectividad", sobre la probabilidad de desertar definitivamente en cada uno de los tramos analizados, al comparar con la categoría de referencia ("Privadas Selectivas").

$\mathrm{Al}$ analizar la interacción entre el tipo de universidad según su selectividad y el quintil de ranking de notas en el establecimiento de origen del estudiante, se aprecian diferencias estadísticamente significativas al comparar con la categoría de referencia ("Privadas Selectivas"). Las diferencias se acentúan en los quintiles extremos de la distribución. En el caso de las universidades "CRUCH Selectivas", los estudiantes pertenecientes al más alto quintil de ranking ("V") poseen en promedio cerca de un $30 \%$ menos de probabilidad de desertar definitivamente, en comparación a los estudiantes que acceden a una universidad "Privada Selectiva". Por otro lado, para el caso de las universidades "CRUCH No Selectivas", los estudiantes pertenecientes al quintil de menor ranking ("I") poseen cerca de un $20 \%$ más de probabilidad de desertar definitivamente que los estudiantes del mismo quintil que accedieron a una universidad de la categoría de referencia ("Privadas Selectivas").

Este resultado implicaría que los estudiantes de mejor ranking poseen una menor probabilidad de deserción en las universidades más selectivas y, por otro lado, que los estudiantes de menor ranking son más propensos a desertar si accedieron a universidades de la categoría "CRUCH No Selectivas". Sin embargo, estos efectos no son muy consistentes debido a que, al comparar con la categoría "Privadas No Selectivas", no se aprecia una tendencia clara.

$\mathrm{Al}$ analizar los efectos de la interacción entre el tipo de universidad según su selectividad y el nivel de ingreso del núcleo familiar reportado en el SIM- 
CE, se aprecian diferencias estadísticamente significativas para las universidades "CRUCH Selectivas" y para las universidades "Privadas No Selectivas" en el tramo inferior de ingreso, al compararlas con la categoría de referencia (universidades "Privadas Selectivas"). Los estudiantes pertenecientes al tramo de menores ingresos $(<200)$ tienen cerca de un $30 \%$ menos de probabilidad de desertar definitivamente si acceden a una universidad "CRUCH Selectiva" o a una "Privada No Selectiva", que si acceden a una universidad "Privada Selectiva".

$\mathrm{Al}$ analizar a los estudiantes provenientes de familias de mayores ingresos $(>1,800)$, se puede apreciar que aquellos que acceden a universidades "CRUCH Selectivas" poseen cerca de un $40 \%$ menos de probabilidad de desertar definitivamente que los estudiantes del mismo tramo de ingreso que acceden a universidades "Privadas Selectivas". Este efecto puede deberse a que la rentabilidad de una carrera en una universidad de alta selectividad, para los estudiantes de mayores ingresos, sea sustantivamente superior a la rentabilidad de las de universidades de menor selectividad.

Al analizar los efectos de la interacción entre el tipo de universidad según su selectividad y la macroárea de conocimiento de la carrera, se observan diferencias estadísticamente significativas al comparar a las universidades "CRUCH Selectivas" en la macroárea de "Salud", con respecto a la categoría de referencia en la misma macroárea. Se aprecia que los estudiantes que acceden a una carrera de la macroárea de "Salud" en una universidad "CRUCH Selectiva", presentan en promedio cerca de un $80 \%$ menos de probabilidad de desertar definitivamente, que aquellos que acceden a una carrera de la macroárea de "Salud" en una universidad de la categoría "Privadas Selectivas". Este efecto es también significativo al comparar, en la misma macroárea, a las universidades "CRUCH No Selectivas" con la categoría de referencia. Una posible explicación a este efecto es que las carreras de la macroárea de "Salud", tanto en las universidades "CRUCH Selectivas" como en las "CRUCH No Selectivas", respondan de manera distinta a la deserción estudiantil, ya sea por características propias de la malla curricular o por políticas de retención de los planteles educacionales. Otra posible explicación es que las universidades de estas categorías concentren una mayor cantidad de estudiantes de la carrera de Medicina en comparación con las universidades "Privadas Selectivas". Dicha carrera presenta tasas muy bajas de deserción universitaria (menor al $10 \%$ en relación a un $26 \%$ en Ingeniería en Obras Civiles y un $53 \%$ en Derecho ${ }^{12}$ (González, 2005)), por lo

\footnotetext{
${ }^{12}$ Tasa de deserción estimada sin diferenciar deserción temporal de deserción definitiva.
}

que el modelo podría identificar parte de este efecto en la interacción entre "Salud" y las universidades pertenecientes al CRUCH.

Otro efecto que resulta estadísticamente significativo es en la macroárea de "Educación y Humanidades". Los estudiantes que acceden a carreras pertenecientes a esta macroárea en universidades "Privadas No Selectivas", poseen una probabilidad $60 \%$ menor de desertar definitivamente, que aquellos estudiantes que acceden a carreras de "Educación y Humanidades" en universidades "Privadas Selectivas". Este efecto, pese a ser estadísticamente significativo, no resulta coherente ya que las carreras de pedagogía que presentan una mayor tasa de deserción provienen de universidades de la categoría "Privadas No Selectivas".

\section{Efectos de la macroárea de carrera}

En este caso el efecto marginal calculado, es el efecto promedio de la "macroárea de conocimiento de la carrera" sobre la probabilidad de desertar definitivamente en cada uno de los tramos analizados, al comparar con la categoría de referencia ("Ciencias sociales").

Al hacer interactuar la macroárea de conocimiento con el quintil de PSU matemática individual del estudiante, se aprecia una mayor diferencia para los quintiles intermedios que para los extremos al comparar las macroáreas de "Educación y Humanidades" y "Ciencias" con la categoría de referencia ("Ciencias sociales"). En el caso de "Ciencias" se aprecia el efecto más notorio, los estudiantes del segundo quintil de PSU matemática individual presentan un $60 \%$ más de probabilidad de desertar definitivamente que los estudiantes pertenecientes al mismo quintil en la macroárea de "Ciencias Sociales", efecto que va disminuyendo en los quintiles superiores, volviéndose no significativo en el quinto quintil de PSU matemática individual del estudiante. En el caso de la macroárea de "Educación y Humanidades" se aprecian diferencias estadísticamente significativas en los primeros cuatro quintiles, teniendo un efecto negativo sobre la probabilidad de desertar definitivamente, en comparación a los mismos quintiles en la categoría de referencia. Sin embargo, su efecto es menos pronunciado que para el caso de "Ciencias". De este resultado puede interpretarse que el efecto de la macroárea de la carrera sobre la deserción definitiva (en el caso de "Ciencias" y "Educación y Humanidades"), se vuelve poco relevante para los estudiantes "más hábiles", afectando principalmente a aquellos que pertenecen a los quintiles intermedios, sobre todo a aquellos estudiantes que acceden a una carrera de la macroárea de "Ciencias". 
Esto refuerza la idea que la habilidad juega un rol importante en la deserción universitaria de las carreras de la macroárea de "Ciencias", pero que las diferencias entre macroáreas no logran afectar fuertemente la probabilidad de deserción de los estudiantes en los quintiles extremos.

Puede notarse también, que el único efecto estadísticamente significativo en el quinto quintil de PSU matemática individual del estudiante es al comparar la macroárea de "Salud" con la categoría de referencia, presentando entre un $20 \%$ y $30 \%$ menos de probabilidad de desertar definitivamente, los estudiantes del quinto quintil que acceden a una carrera de la macroárea de "Salud", con respecto a "Ciencias Sociales". Nuevamente este efecto podría estar identificando a estudiantes pertenecientes a carreras con una muy baja tasa de deserción en la macroárea de "Salud", como por ejemplo Medicina.

$\mathrm{Al}$ analizar el efecto de la interacción de la macroárea de conocimiento con el quintil de ranking de notas del estudiante en el establecimiento de origen, se aprecian efectos estadísticamente significativos en las macroárea de "Salud" y "Educación y Humanidades". Puede observarse una leve tendencia a la baja en la probabilidad de desertar definitivamente con respecto a la categoría de referencia, pasando de no ser estadísticamente significativa la comparación en el quintil de menor ranking ("I"), a presentarse una mayor divergencia en el quinto quintil. Los estudiantes del mayor quintil de ranking de notas ("V") poseen entre un $30 \%$ y un $40 \%$ menos de probabilidad de desertar definitivamente, si acceden a carreras de la macroárea de "Salud" o de "Educación y Humanidades", en comparación a "Ciencias Sociales". Por otro lado no se aprecia heterogeneidad al analizar la macroárea de "Ciencias" en comparación a "Ciencias Sociales".

Este resultado implicaría que el efecto de la macroárea (en el caso de "Salud" y "Educación y Humanidades"), se acentúa en el quintil de mayor ranking de notas, siendo menos relevante en el quintil de menor ranking de notas.

$\mathrm{Al}$ analizar la interacción entre macroáreas de conocimiento y el nivel de ingreso del núcleo familiar según el SIMCE, se aprecian diferencias estadísticamente significativas en la macroárea de "Ciencias" con respecto a la categoría de referencia ("Ciencias Sociales") para los cuatro primeros tramos de ingreso. Al analizar el efecto de las macroáreas en los últimos tramos de ingreso, no se aprecian diferencias estadísticamente significativas, por lo que el efecto de la macroárea sobre la deserción se atenúa al considerar los tramos de mayor ingreso socioeconómico. El nivel de ingreso afecta más la deserción definitiva en las carreras de la macroárea de
"Ciencias", posiblemente, debido a un mayor costo promedio del arancel de carrera dentro de la macroárea de "Ciencias", que en el resto de las macroáreas. En particular, los estudiantes del tramo de menores ingresos $(<200)$ poseen en promedio un $40 \%$ más de probabilidad de desertar definitivamente, si acceden a una carrera de la macroárea de "Ciencias", que si acceden a una carrera de la macroárea de "Ciencias Sociales".

Cabe notar que existen también diferencias estadísticamente significativas para los primeros tramos de ingreso, al comparar las macroáreas de "Salud" y "Educación y Humanidades" con la categoría de referencia. Si bien esta se vuelve estadísticamente no significativa en los tramos de mayor ingreso, esto es debido principalmente al aumento de los intervalos de confianza y no a la estimación puntual del efecto, por lo que no se infiere una tendencia en estos casos.

Al analizar la interacción entre macroáreas de conocimiento y los años de acreditación de la universidad, se aprecian efectos heterogéneos para la macroárea de "Educación y Humanidades" (identifica sobre todo a carreras de pedagogía) con respecto a la categoría de referencia ("Ciencias sociales"). En las carreras de "Educación y Humanidades" en las universidades no acreditadas, se observa una mayor probabilidad de deserción definitiva que en las carreras pertenecientes a la macroárea de "Ciencias Sociales", del mismo grupo de universidades. Los estudiantes que acceden a una carrera de la macroárea de "Educación y Humanidades" en una universidad "No Acreditada", presentan cerca de un $60 \%$ más de probabilidad de desertar definitivamente, que los estudiantes que acceden a universidades en la misma categoría de acreditación en la macroárea de "Ciencias Sociales". Sin embargo, este efecto se invierte al analizar las carreras pertenecientes a universidades acreditadas entre $6 \mathrm{y}$ 7 años. Los estudiantes que acceden a una carrera de la macroárea de "Educación y Humanidades" en una universidad acreditada entre 6 y 7 años, presentan cerca de un $40 \%$ menos de probabilidad de desertar definitivamente, que los estudiantes que acceden a universidades en la misma categoría de acreditación en la macroárea de "Ciencias Sociales". Este resultado tiene múltiples interpretaciones, una posible explicación es que la calidad de las carreras de pedagogía cambia drásticamente de universidades no acreditadas a universidades acreditadas entre 6 y 7 años, en comparación al cambio de calidad de las carreras de "Ciencias Sociales" entre estos dos grupos de universidades. Un aumento de calidad en esta macroárea puede traducirse en una disminución mayor de la deserción definitiva, en comparación con la macroárea de "Ciencias Sociales". Otra explicación, complementaria a la an- 
terior, es que el nivel de ingreso futuro esperado para las carreras de "Educación y Humanidades" en estos dos grupos de universidades, varíe en una mayor proporción que para las carreras de "Ciencias Sociales". Esto se traduciría en que la tasa de retorno del estudiante de seguir en el sistema universitario, aumentaría en una mayor proporción en las carreras de "Educación y Humanidades" que en las carreras de "Ciencias Sociales", comparando carreras de universidades no acreditadas con carreas de universidades acreditadas entre 6 y 7 años.

\section{Conclsiones e implicancias de política pública}

Utilizando un sistema de tres ecuaciones simultáneas, se logró modelar el fenómeno de la deserción universitaria, considerando que la decisión de acceder al sistema universitario puede afectar la decisión posterior de desertar, permitiendo la correlación de la heterogeneidad no observable. Por otro lado, el integrar en el proceso de decisión la opción de desertar temporalmente diferenciada de la de desertar definitivamente, permitió obtener efectos más precisos sobre la deserción definitiva del sistema universitario, sin confundir categorías ni perder observaciones ex ante.

La muestra utilizada permitió realizar un análisis sobre la deserción en el sistema universitario en su conjunto, encontrándose tasas del $16 \%$ de Deserción definitiva y $12 \%$ de Deserción temporal, alcanzándose cerca de un $29 \%$ de deserción general. Ambos procesos ocurren principalmente los dos primeros años de universidad, por lo que las variables de acceso son determinantes. Por otro lado, la deserción temporal está estrechamente ligada al cambio de carrera y universidad, lo que da cuenta de problemas en la movilidad de los estudiantes al interior del sistema.

Existe un problema de endogeneidad presente en las ayudas estudiantiles, el cual afecta directamente la estimación de su efecto sobre la deserción universitaria. Utilizando un enfoque de variable instrumental, similar al propuesto por Meneses y Blanco (2010), se logró corregir en parte esta endogeneidad, obteniendo que las ayudas estudiantiles disminuyen la probabilidad de desertar definitivamente en más de un $60 \%$. Este efecto es cerca de un $20 \%$ mayor al calculado sin corregir la endogeneidad. Por otro lado, el efecto de la selección de muestra no resultó estadísticamente significativo de manera directa, lo que indicaría que la población de la cohorte analizada que accede a la universidad es bastante representativa de la población potencial (estudiantes que rinden la PSU en el año 2005 o en el año 2006). Sin embargo, al considerar también el efecto de las ayudas estudiantiles en el acceso a la universidad, existe un efecto indirecto sobre la estimación de esta variable, el cual modifica su estimación y la de variables correlacionadas como el nivel de ingreso del núcleo familiar. Si bien la corrección de endogeneidad resultó estadísticamente significativa, tanto de manera directa como indirecta, a medida que la especificación del modelo se vuelve más robusta, la corrección resulta menor. Esto indica que la omisión de variables relevantes puede afectar en mayor medida la estimación insesgada de las variables del modelo, que la inclusión de una variable de naturaleza endógena o la selección de muestra en el caso de la deserción universitaria.

Se pudo constatar la correlación estadísticamente significativa de distintos conjuntos de variables: habilidad individual del estudiante, factores socioeconómicos y demográficos a nivel individual y del núcleo familiar, características del establecimiento de origen, la calidad de la universidad a la cual accede el estudiante, el tipo de carrera a la cual accede el estudiante y factores relacionados con la proyección laboral futura.

El puntaje NEM y el ranking de notas de enseñanza media son factores explicativos de la habilidad del estudiante mucho más relevantes que los puntajes en la PSU de matemática y lenguaje. En este sentido, la "habilidad" que estaría midiendo la PSU no sería tan relevante en el fenómeno de la deserción universitaria.

En relación a las características socioeconómicas y demográficas, los factores que emergen con mayor relevancia son: el nivel ingreso económico del núcleo familiar y si el estudiante es beneficiado con una ayuda estudiantil, ya sea crédito, becas, o una combinación de estos. En un menor grado afecta el nivel educacional alcanzado por la madre del estudiante, la edad y el género. Estudiantes de mayores ingresos poseen una menor probabilidad de desertar que aquellos estudiantes de familias con menores ingresos, sin embargo, aquellos estudiantes que perciben una ayuda estudiantil poseen también una menor probabilidad de desertar que aquellos que no perciben beneficio alguno. En este sentido la deserción universitaria se configura también como un problema de financiamiento.

Otro grupo de factores que afecta fuertemente la deserción universitaria son los relacionados con la calidad del establecimiento de origen. Tanto el puntaje de PSU matemática del establecimiento de origen, como el nivel de vulnerabilidad económica medido por el IVE son factores determinantes. Los estudiantes provenientes de colegios con mejores pun- 
tajes en la PSU de matemática y que poseen un menor grado de vulnerabilidad económica, presentan una menor probabilidad de deserción, incluso al controlar por la habilidad individual del estudiante. Esto da cuenta que existen ciertos factores relacionados con la calidad del establecimiento de origen del estudiante, que afectan fuertemente la deserción universitaria y que no son capturados por la habilidad individual. Una posible explicación, es que los estudiantes provenientes de mejores establecimientos poseen una mejor red de apoyo, o bien, que en los establecimientos de más altos puntajes y menos vulnerables, los estudiantes desarrollen competencias que mejoren considerablemente su desempeño en la universidad.

Si bien los factores previos al acceso son determinantes significativos de la deserción, también influyen en este fenómeno las características de la carrera y la universidad a la cual se accede. Los estudiantes que acceden a universidades de mejor calidad presentan una menor probabilidad de desertar del sistema universitario que aquellos que acceden a universidades de peor calidad. Las variables que emergen como mejores predictores de la deserción universitaria, en este caso, son el puntaje promedio de PSU de los estudiantes que acceden a la universidad y si esta está acreditada o no, perdiendo relevancia el tipo de universidad según su selectividad. Por otro lado, en relación con las variables que identifican a la carrera que accede el estudiante, se muestran diferencias estadísticamente significativas por macroárea de conocimiento, presentando una mayor probabilidad de deserción los estudiantes que acceden a carreras de "Ciencias" y una menor probabilidad de deserción aquellos que acceden a carreras de "Educación y Humanidades". Estos resultados resaltan la importancia de analizar efectos por medio de modelos formales, ya que la estadística descriptiva presenta tasas de deserción por macroárea que podría llevar a conclusiones erradas.

Siguiendo los modelos de Bean (1980) y St.John et al. (2000), otro factor determinante de la deserción universitaria está relacionado con la realidad del mercado laboral que podría enfrentar el estudiante. En este sentido, la probabilidad de encontrar empleo resulta estadísticamente significativa y sustantiva para disminuir la deserción universitaria. Los estudiantes que acceden a carreras con mejor empleabilidad poseen una menor probabilidad de desertar que aquellos que acceden a carreras de baja empleabilidad.

Realizando un análisis más detallado en relación al tipo de universidad y carrera que accede el estudiante, se pudo constatar que existe cierto grado de heterogeneidad de algunos factores. Tanto el nivel de ingreso como el ranking de notas del estudiante en su establecimiento de origen, responden de distinta forma en cada grupo de universidades, existiendo diferencias en las categorías extremas. Los estudiantes de mayores ingresos y los de mejor ranking de notas, poseen una probabilidad estadísticamente significativa menor de desertar definitivamente si accedieron a universidades selectivas del CRUCH, que aquellos que accedieron a universidades de menor selectividad.

En relación a la carrera, la habilidad individual medida por la prueba PSU de matemática, el nivel de ingreso del núcleo familiar, el ranking de notas y la acreditación de la universidad, interactúan con la macroárea de conocimiento de la carrera a la cual accede el estudiante. En este caso la habilidad medida por medio de la PSU de matemática, afecta de mayor manera a los estudiantes que acceden a carreras de "Ciencias", presentando una mayor probabilidad de desertar definitivamente en los quintiles intermedios de la distribución. Por otro lado, el ranking de notas del estudiante en el establecimiento de origen afecta en mayor medida a las carreras de "Salud" y "Educación y Humanidades", presentando probabilidades de Deserción definitiva menores y estadísticamente significativas en el quintil de mayor ranking de notas. El nivel de ingreso afecta mayormente a las carreras de "Ciencias" que a las demás macroáreas de conocimiento. Los estudiantes pertenecientes a los tramos de menor ingreso presentan una probabilidad estadísticamente significativa mayor de desertar definitivamente que aquellos que acceden a otras macroáreas, sin embargo, esta diferencia se atenúa en los tramos de mayor ingreso.

Otro resultado a destacar, es que existe una heterogeneidad considerable dentro de las macroáreas de las carreras. En el caso de "Educación y Humanidades" se concluye que la relación con la deserción cambia drásticamente al comparar carreras no acreditadas con carreras acreditadas entre 6 y 7 años. Esto evidencia las diferencias de calidad existentes entre las carreras de pedagogía de universidades no acreditadas, con las universidades de mayor excelencia.

Finalmente se concluye que la deserción universitaria es influenciada por diversos tipos de factores. Algunos de estos factores son relacionados con las características personales del estudiante y de su núcleo familiar, sin embargo, cobran una gran relevancia aquellos factores relacionados con la calidad del establecimiento de origen del estudiante, con la calidad de la universidad y con el tipo de carrera a la cual se accede.

Al finalizar este estudio emerge como posible extensión, considerar el proceso de cambio de carrera o universidad en el fenómeno de la deserción uni- 
versitaria y relajar el orden impuesto entre Deserción temporal y Deserción definitiva (en la función de utilidad). Para ello puede utilizarse un modelo de duración en el tiempo, como el propuesto por DesJardins et al. (2006), pero aplicado a todo el sistema universitario. De esta forma podría estimarse la causalidad de ciertas variables de interés, como las becas y créditos, sin la necesidad de construir instrumentos exógenos y, además, comprender la real influencia del tiempo y la ocurrencia de ciertos eventos en la decisión de desertar del sistema universitario, cambiar de carrera o universidad o continuar sin cambios en el sistema. Para ello es necesario ampliar la información ya recopilada, incluyendo las variaciones en el tiempo de las variables a estudiar.

Debido a la multicausalidad del fenómeno de la deserción, para abordarlo de manera correcta es necesario un enfoque sistémico. Algunas ideas que podrían ayudar a la disminución de la deserción en el sistema universitario son el mejorar la calidad de los establecimientos de educación secundaria, asegurando además la calidad de la carrera y universidad a la cual accede el estudiante; aumentar el financiamiento por medio de becas y créditos; mejorar los instrumentos de selección universitaria; promover la realización de programas de retención a nivel carreras y casas de estudio y mejorar la movilidad de los estudiantes al interior del sistema.

El fenómeno de la deserción universitaria es afectado fuertemente por características ajenas al estudiante y su núcleo familiar. La calidad, tanto del establecimiento de origen como de la universidad y carrera a la cual accede el estudiante, son factores altamente influyentes en la deserción universitaria. En este sentido, el aseguramiento de la calidad de las instituciones de educación pareciera ser fundamental para abordar este problema.

Luego de constatarse un efecto positivo y estadísticamente significativo de las ayudas estudiantiles en la disminución de la deserción universitaria, la entrega de becas y créditos iría en la dirección correcta para mejorar la retención y disminuir las elevadas tasas de deserción, tanto temporal como definitiva. Claramente este estudio no logra diferenciar si este efecto varía al percibir sólo becas o solo créditos, por lo que es necesario ahondar en las diferencias entre estos dos tipos de instrumentos.

En relación al sistema de selección a la educación superior, se aprecia que ciertos factores tienen un mayor impacto en la deserción universitaria. Tanto el puntaje NEM como el ranking de notas parecieran ser más relevantes que los puntajes de PSU matemática y lenguaje, por lo que esto debe ser considerado al decidir las ponderaciones de cada factor para acceder a las distintas universidades. En este sentido, la inclusión del ranking en el sistema de selección no debería ir en desmedro del puntaje NEM, ya que ambos factores están correlacionados con la disminución de la deserción universitaria. Esta medida permitiría mejorar la equidad del sistema, teniendo en cuenta también el problema de la deserción.

Si bien las políticas transversales a nivel del sistema universitario son muy importantes para combatir la deserción, también es necesario abordar la heterogeneidad presente en cada tipo de universidad y carrera. Los estudiantes responden de distinta forma a la deserción universitaria dependiendo del tipo de carrera y universidad que eligen, por lo que es necesaria la implementación de políticas que aborden el problema a nivel de universidades y áreas de carrera. Por ello se propone la realización de programas de retención a nivel de universidad y áreas, que identifiquen los grupos de riesgo y aborden las características particulares de las distintas carreras y casas de estudio. La decisión de desertar o no, está también ligada a las características personales del estudiante, por lo que el apoyo a nivel personal resulta fundamental.

Otro aspecto a considerar es la calidad de la información que reciben los estudiantes y su movilidad al interior del sistema universitario. Al diferenciar la deserción temporal de la definitiva pudo constatarse que el fenómeno temporal está estrechamente ligado al cambio de carrera y universidad. Cerca del $80 \%$ de los estudiantes que desertan temporalmente cambian de carrera o universidad. En este sentido, la deserción temporal del sistema puede deberse a que los estudiantes eligieron erróneamente la carrera a la cual accedieron inicialmente, ya sea por falta de información o por problemas de índole vocacional, siendo fundamental proporcionar información fidedigna sobre las distintas carreras universitarias, las mallas curriculares y la proyección laboral del egresado, además de promover la orientación vocacional en la educación secundaria y durante el desarrollo de la carrera universitaria.

Además de mejorar la información sobre las carreras y universidades y promover la orientación vocacional antes y durante la carrera universitaria, se podría contribuir a que los estudiantes puedan tener una mejor movilidad al interior del sistema, sin la necesidad de ausentarse por uno o dos años para poder cambiar de institución o carrera. En esta línea, el validar el puntaje PSU por dos años pareciera ser una buena medida, sin embargo, es importante también realizar esfuerzos en el reconocimiento curricular entre distintas instituciones y carreras, para permitir disminuir los retrasos en los procesos de titulación. 
Finalmente, el problema de la deserción universitaria debe ser abordado desde distintos focos, ya que es un fenómeno en el cual afectan e interactúan distintos factores. Es necesario desarrollar investigaciones que permitan comprender de mejor manera este problema, evaluando distintas medidas que ayuden a la disminución de las elevadas tasas de deserción en el sistema universitario chileno.

\section{Referencias}

Acuña, C., Makovec, M., y Mizala, A. (2010). Access to higher education and the probability of drop-out: evidence from a cohort of Chilean secondary school leavers.

Bank Slaving, R. \& Biddke, B., B. (1990). Effects of Peer, Faculty and Parental Influences on Students Persistence. Sociology Of Education, (63):208-225.

Barrios F., A. (2011). Deserción universitaria en Chile: incidencia del financiamiento y otros factores asociados. revista CIS, (14):59-72.

Bean, J. P. (1980). Dropouts and turnover.The synthesis and test of a causal model of student attrition. Research in Higher Education, 2(12):155187.

Becker, G. (1964). Human Capital: A theoretical and empirical analysis with special reference to education. National Bureau of Economic Research, New York.

Bratti, M. y Miranda, A. (2009). Selection endogenous dummy ordered probit and selection endogenous dummy dynamic ordered probit models. Munich Personal Repec Archive, forthcoming.

Bratti, M. y Miranda, A. (2010). Non-pecunary returns to higher education: the effecto on smoking intensisy in the UK. Health Economics, (19):906920.

Cameron, L. (2009). Can a public scholarship program successfully reduce school drop-outs in a time of economic crisis? Evidence from Indonesia. Economics of Education Review, (28):308-317.

Centro de microdatos (2008). Informe final: "Estudios sobre las causas de la deserción universitaria". Universidad de Chile, Departamento de Economía.

Comisión de Financiamiento Estudiantil para la Educación Superior (2012). Andálisis y recomendaciones para el sistema de financiamiento estudiantil. Santiago de Chile.
DesJardins, S. L., Ahlburg, D. A., y McCal, B. P. (2006). The effects of interrupted enrollment on graduation from college: Racial, income, and ability differences. Economics of Education Review, (25):575-590.

Díaz, C. P. (2008). Modelo conceptual para la deserción estudiantil universitaria chilena. Estudios Pedagógicos, XXXIV(2):65-86.

Donoso, P. y Schiefelbein, E. (2007). Análisis de los modelos explicativos de retención de estudiantes en la universidad: una visión desde la desigualdad social. Estudios Pedagógicos, XXXIII(1):7-27.

González, L. y Uribe, D. (2002). Estimaciones sobre la "repitencia" y deserción superior chilena. Consideraciones sobre sus implicaciones. Revista de la educación, (17):251-267.

González, L. E. (2005). Estudio sobre la repitencia y deserción en la educación superior chilena. Technical report, Santiago.

Heckman, J. J. (1979). Sample Selection Bias as a Specification Error. Econometrica, 47(1):153161 .

Himmel, E. (2002). Modelos de Análisis de la Deserción Estudiantil en la. Revista Calidad en La Educación, pp. 17,75-90.

Humlum, M. K., Kleinjans, K. J., y Nielsen, H. S. (2007). An Economic Analysis of Identity and Career Choice.

Intelis y Verde (2012). Evaluación de impacto de las becas de educación superior del MINEDUC. EconomÃa y Negocios, Universidad de Chile, Santiago de Chile.

Jalan, J. y Ravallion, M. (2003). Does piped water reduce diarrhea for children in rural India? Journal of Econometrics, (112):153-173.

Lassibille, G. y Navarro Gómez, L. (2008). Why do higher education students drop out? Evidence from Spain. Education Economics, 16(1):89-105.

Melguizo, T., Sanchez Torres, F., y Jaime, H. (2011). The association between financial aid availability and the college drop out rates in Colombia. Higher Education, (62):231-247.

Meller, P. y Lara, B. (2010). Carreras universitarias: rentabilidad, selectividad y discriminación. Centro de Investigación Avanzada en Educación, Universidad de Chile.

Meneses, F. y Blanco, C. (2010). Financial Aid and Higher Education Enrollment in Chile: A Government Policy Analysis. 
MINEDUC (2012). Deserción en la educación superior en Chile. Serie Evidencias.

Miranda, A. y Rabe-Hesketh, S. (2005). Estimation of ordinal response models, accounting for sample selection bias. En 11th UK Stata Users Group meeting proceedings.

Rau, T., Rojas, E., y Urzúa, S. (2012). Higher Education Dropouts, Access to Credit, and Labor Market Outcomes: Evidence from Chile.

Roberts, M. R. y Whited, T. M. (2012). Endogeneity in Empirical Corporate Finance.

Roodman, D. (2009). Estimating Fully Observed Recursive Mixed-Process Models with cmp.

Solis, A. (2011). Credit Access and College Enrollment.

St.John, E. P., Cabrera, A. F., Nora, A., y Asker, E. H. (2000). Economic influences on persistence reconsidered. In J. M. Braxton (Ed.). En Reworking the student departure puzzle, pp. 29-47. Vanderbilt University Press, Nashville.

Stratton, L. S., O'Toole, D. M., y Wetzel, J. N. (2008). A multinomial logit model of college stopout and dropout behavior. Economics of Education Review, (27):319-331.

Tinto, V. (1975). Dropout From Higher Education: A Theoretical Synthesis of Recent Research. Review of Educational Research, (45):89-125.

UNESCO (2011). Clasificación Internacional Normalizada de la Educación (CINE 2011). Technical report, Instituto de Estadística, Organización de las Naciones Unidas para la Educación, la Ciencia y la Cultura. 